C. P. Boyer, E. G. Kalnins, and W. Miller, Jr.

Nagoya Math. J.

Vol. 60 (1976), $35-80$

\title{
SYMMETRY AND SEPARATION OF VARIABLES FOR THE HELMHOLTZ AND LAPLACE EQUATIONS
}

\author{
C. P. BOYER, E. G. KALNINS, AND W. MILLER, JR.
}

\section{Introduction.}

This paper is one of a series relating the symmetry groups of the principal linear partial differential equations of mathematical physics and the coordinate systems in which variables separate for these equations. In particular, we mention [1] and paper [2] which is a survey of and introduction to the series. Here we apply group-theoretic methods to study the separable coordinate systems for the Helmholtz equation.

$$
\begin{gathered}
\left(\Delta_{3}+\omega^{2}\right) \Psi(\boldsymbol{x})=0, \quad \boldsymbol{x}=\left(x_{1}, x_{2}, x_{3}\right), \\
\Delta_{3}=\partial_{11}+\partial_{22}+\partial_{33}, \quad \omega>0,
\end{gathered}
$$

and the Laplace equation

$$
\Delta_{3} \Psi(x)=0 .
$$

It is well-known that (0.1) separates in eleven coordinate systems, see [3], Chapter 5, and references contained therein. Moreover, in [4] it is shown that these systems correspond to commuting pairs of second order symmetric operators in the enveloping algebra of $\mathscr{E}(3)$, the symmetry algebra of (0.1). However, we show here for the first time how one can systematically make use of the representation theory of the Euclidean symmetry group $E(3)$ of the Helmholtz equation to derive identities relating the different separable solutions. As we will point out, some of these identities are new.

It is also known that there are 17 types of cyclidic coordinate systems which permit $R$-separation of variables in the Laplace equation and these appear to be the only such separable systems for (0.2), [5]. (An $R$ separable coordinate system $\{u, v, w\}$ for $(0.2)$ is a coordinate system which permits a family of solutions

Received August 18, 1975. 


$$
\Psi_{\lambda, \mu}(\boldsymbol{x})=R(u, v, w) U_{\lambda, \mu}(u) V_{\lambda, \mu}(v) W_{\lambda, \mu}(w)
$$

where $\lambda, \mu$ are the separation constants and $R$ is a fixed factor such that either $R \equiv 1$ (pure separation) or $R \neq \equiv 1$ and $R$ cannot be written in the form $R=R_{1}(u) R_{2}(v) R_{3}(w)$.) In Sections 1 and 6 we will list all of these systems together with the $R$-separable solutions and show for the first time that each system corresponds to a commuting pair of second-order symmetric operators in the enveloping algebra of the conformal symmetry algebra $s o(4,1)$ of $(0.2)$. Furthermore, we will demonstrate how to use the local representation theory of the conformal group to derive identities relating the various separable solutions of the Laplace equation.

In order to make this paper more useful for the reader interested in applications of the representation-theoretic characterization of separation of variables to other problems we have listed the separation equations and separated solutions in some detail.

Finally, we point out that this work is very much in the spirit of the books of Harry Bateman, [6], [7], who earlier showed the great importance of separation of variables for the derivation of physically significant special function identities.

Section 1. The symmetry groups of the Helmholtz and Laplace equations.

A symmetry operator for (0.1) is a linear differential operator

$$
L=\sum_{j=1}^{3} a_{j}(\boldsymbol{x}) \partial_{j}+b(\boldsymbol{x})
$$

where $a_{j}, b$ are analytic functions of $x_{1}, x_{2}, x_{3}$ in some domain $\mathscr{D}$ in $\boldsymbol{R}^{3}$ such that $L \psi$ is a solution of the Helmholtz equation in $\mathscr{D}$ for any analytic solution $\psi$ of $(0.1)$ in $\mathscr{D},[2]$. It is easy to show that the set of all such symmetry operators is a Lie algebra under the operations of scalar multiplication and commutator bracket $\left[L_{1}, L_{2}\right]=L_{1} L_{2}-L_{2} L_{1}$. In particular, apart from the trivial symmetry $E=1$, the symmetry algebra of $(0.1)$ is six-dimensional with basis

$$
\begin{gathered}
P_{j}=\partial_{j}, \quad j=1,2,3, \\
J_{1}=x_{3} \partial_{2}-x_{2} \partial_{3}, J_{2}=x_{1} \partial_{3}-x_{3} \partial_{1}, J_{3}=x_{2} \partial_{1}-x_{1} \partial_{2},
\end{gathered}
$$

and commutation relations

$$
\begin{gathered}
{\left[J_{\ell}, J_{m}\right]=\sum_{n} \varepsilon_{\ell m n} J_{n}, \quad\left[J_{\ell}, P_{m}\right]=\sum_{n} \varepsilon_{\ell m n} P_{n}} \\
{\left[P_{\ell}, P_{m}\right]=0, \quad \ell, m, n=1,2,3,}
\end{gathered}
$$


where $\varepsilon_{\ell m n}$ is the completely skew-symmetric tensor such that $\varepsilon_{123}=+1$. We take the real Lie algebra $\mathscr{E}(3)$ with basis (1.2) as the symmetry algebra of (0.1). In terms of the $P$-operators the Helmholtz equation reads

$$
\left(P_{1}^{2}+P_{2}^{2}+P_{3}^{2}\right) \psi=-\omega^{2} \psi,
$$

and each element of $\mathscr{E}(3)$ commutes with $\sum_{j=1}^{3} P_{j}^{2}$.

As is well-known, $\mathscr{E}(3)$ is isomorphic to the Lie algebra of the Euclidean group in three-space $E(3)$ and the subalgebra $s o(3)$ with basis $\left\{J_{1}, J_{2}, J_{3}\right\}$ is isomorphic to the Lie algebra of the proper rotation group $S O(3)$, [8]. Indeed, consider the well-known realization of $S O(3)$ as the group of real $3 \times 3$ matrices $A$ such that $A^{t} A=E_{3}$ and $\operatorname{det} A=+1$. (Here, $E_{3}$ is the $3 \times 3$ identity matrix.) The Lie algebra of $S O(3)$ in this realization is the space of $3 \times 3$ skew symmetric matrices $\mathscr{A}$, $\left(\mathscr{A}^{t}=-\mathscr{A}\right)$. A basis for this Lie algebra is provided by the matrices

$$
\mathscr{J}_{1}^{\prime}=\left(\begin{array}{rrr}
0 & 0 & 0 \\
0 & 0 & -1 \\
0 & 1 & 0
\end{array}\right), \quad \mathscr{J}_{2}^{\prime}=\left(\begin{array}{rrr}
0 & 0 & 1 \\
0 & 0 & 0 \\
-1 & 0 & 0
\end{array}\right), \quad \mathscr{J}_{3}^{\prime}=\left(\begin{array}{rrr}
0 & -1 & 0 \\
1 & 0 & 0 \\
0 & 0 & 0
\end{array}\right)
$$

with commutation relations $\left[\mathscr{J}_{\ell}^{\prime}, \mathscr{J}_{m}^{\prime}\right]=\sum_{n} \varepsilon_{\ell m n} \mathscr{J}_{n}^{\prime}$ in agreement with (1.3). A convenient parametrization of $S O(3)$ is that in terms of Euler angles $(\varphi, \theta, \psi)$ :

$$
\begin{gathered}
A(\varphi, \theta, \psi)=\exp \left(\varphi \mathscr{J}_{3}^{\prime}\right) \exp \left(\theta \mathscr{J}_{1}^{\prime}\right) \exp \left(\psi_{\mathscr{J}_{3}^{\prime}}^{\prime}\right), \\
0 \leq \varphi \leq 2 \pi, 0 \leq \theta \leq \pi, 0 \leq \psi \leq 2 \pi,
\end{gathered}
$$

see [8] for details.

The Euclidean group in three-space $E(3)$ can be realized as a group of $4 \times 4$ real matrices

$$
g(A, \boldsymbol{a})=\left(\begin{array}{c:c}
A & 0 \\
& 0 \\
\hdashline a_{1} a_{2} a_{3} & 1
\end{array}\right), \quad \begin{aligned}
& \boldsymbol{a}=\left(a_{1}, a_{2}, a_{3}\right) \in \boldsymbol{R}^{3}, \\
& \text {. }
\end{aligned}
$$

with group product given by matrix multiplication:

$$
g(A, \boldsymbol{a}) g\left(A^{\prime}, \boldsymbol{a}^{\prime}\right)=g\left(A A^{\prime}, \boldsymbol{a} A^{\prime}+\boldsymbol{a}^{\prime}\right) .
$$

Now $E(3)$ acts as a transformation group in three-space $\boldsymbol{R}^{3}$. The group element $g(A, a)$ maps the point $\boldsymbol{x} \in \boldsymbol{R}^{3}$ to the point 


$$
x g=x A+a \in R^{3} .
$$

It follows easily from this definition that $\boldsymbol{x}\left(g g^{\prime}\right)=(\boldsymbol{x} g) g^{\prime}$ for all $\boldsymbol{x} \in \boldsymbol{R}^{3}$, $g, g^{\prime} \in E(3)$ and that $\boldsymbol{x} g\left(E_{3}, \boldsymbol{O}\right)=\boldsymbol{x}$. Geometrically, $g$ corresponds to a rotation $A$ about the origin $\boldsymbol{O} \in \boldsymbol{R}^{3}$ followed by a translation $\boldsymbol{a}$.

A basis for the Lie algebra of the matrix group $E(3)$ is given by the matrices

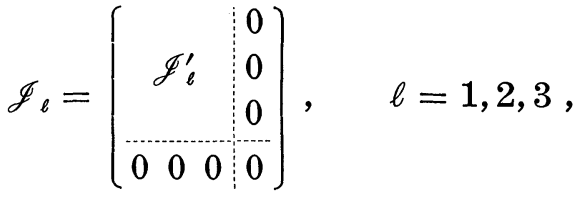

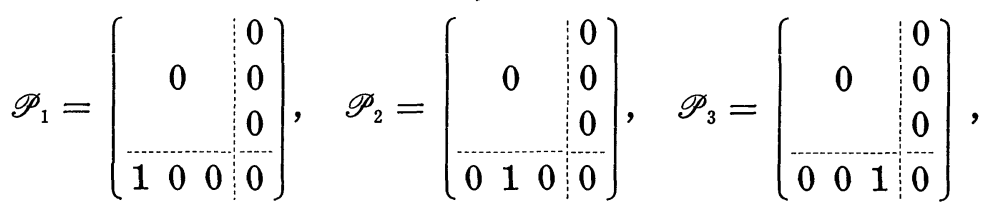

with commutation relations identical to (1.3). This shows that the Lie algebra $\mathscr{E}(3)$ with basis (1.2) is isomorphic to the Lie algebra of $E(3)$. The explicit relation between the Lie algebra generators (1.10) and the group elements (1.7) is

$$
\begin{aligned}
g(\varphi, \theta, \psi, \boldsymbol{a}) & \equiv g(A(\varphi, \theta, \psi), \boldsymbol{a}) \\
& =A(\varphi, \theta, \psi) \exp \left(a_{1} \mathscr{P}_{1}+a_{2} \mathscr{P}_{2}+a_{3} \mathscr{P}_{3}\right)
\end{aligned}
$$

where $A$ is given by (1.6).

Using standard Lie theory methods we can extend the action of $\mathscr{E}(3)$ by Lie derivatives (1.2) on the space $\mathscr{F}$ of analytic functions defined on some open connected set $\mathscr{D} \subseteq R^{3}$, to a local representation $\boldsymbol{T}$ of $E(3)$ on $\mathscr{F},[9]$. We find

$$
\begin{aligned}
\boldsymbol{T}(g) \Phi(\boldsymbol{x})= & \left\{\exp \left(\varphi J_{3}\right) \exp \left(\theta J_{1}\right) \exp \left(\psi J_{3}\right)\right. \\
& \left.\times \exp \left(a_{1} P_{1}+a_{2} P_{2}+a_{3} P_{3}\right)\right\} \Phi(x)=\Phi(\boldsymbol{x} g)
\end{aligned}
$$

where $x g$ is defined by (1.9). Here,

$$
\exp (a L)=\sum_{n=0}^{\infty} a^{n} L^{n} / n !
$$

and $\Phi \in \mathscr{F} . \quad$ By construction

$$
T\left(g g^{\prime}\right)=T(g) T\left(g^{\prime}\right), \quad g, g^{\prime} \in E(3),
$$


and the operators $\boldsymbol{T}(g)$ map solutions of the Helmholtz equation to solutions.

In a manner analogous to the construction of the first-order symmetry operators $L,(1.1)$, we can also determine the possible second-order differential operators $S$ which map solutions of (0.1) into solutions. Let $\mathscr{S}$ be the vector space of all such operators. Among the elements of $\mathscr{S}$ are the trivial symmetries $\Phi Q, \Phi \in \mathscr{F}, Q=\sum_{j=1}^{3} P_{j}^{2}+\omega^{2}$. (Clearly $\Phi Q$ is a symmetry operator since it maps every solution of (0.1) to the zero solution.) Factoring out the subspace $\mathscr{Q}$ of trivial symmetries, one can show by a tedious computation that $\mathscr{S} / \mathscr{Q}$ is forty-one dimensional with a basis consisting of the identity operator $E$, the six first-order operators $J_{\ell}, P_{\ell}$ and thirty-four purely second-order symmetrized operators. The space $\mathscr{E}(3)^{2}$ of second-order symmetrized operators is spanned by the elements $\left\{J_{\ell}, J_{m}\right\},\left\{J_{\ell}, P_{m}\right\},\left\{P_{\ell}, P_{m}\right\}$ where

$$
\{A, B\}=A B+B A,
$$

and these elements are subject only to the relations

$$
\begin{gathered}
\boldsymbol{J} \cdot \boldsymbol{P}=\sum_{k=1}^{3} J_{k} P_{k} \equiv 0, \\
\boldsymbol{P} \cdot \boldsymbol{P}=\sum_{k=1}^{3} P_{k}^{2}=-\omega^{2},
\end{gathered}
$$

the latter relation holding only on the solution space of $(0.1)$, [4].

Since (0.1) is an equation in three variables, two separation constants are associated with each separable coordinate system. Based on the general program relating symmetry to separation of variables, [2], we expect the separated solutions for orthogonal coordinate systems to be characterized as common eigenfunctions of a pair of commuting symmetry operators in $\mathscr{E}(3)^{2}$. This was shown to be the case already in [4]. (In addition one can find a number of rather trivial nonorthogonal separable coordinate systems which correspond to the diagonalization of first-order operators.) The authors of this work showed that each of the eleven orthogonal separable systems corresponds to a pair of independent commuting operators $S_{1}, S_{2}$ in $\mathscr{E}(3)^{2}$ such that the associated separable solutions $\psi=U(u) V(v) W(w)$ are characterized by the eigenvalue equations

$$
\left(\Delta_{3}+\omega^{2}\right) \psi=0, \quad S_{1} \psi=\omega_{1}^{2} \psi, S_{2} \psi=\omega_{2}^{2} \psi
$$


where $\omega_{1}^{2}, \omega_{2}^{2}$ are the separation constants. (It can be shown that there are no non-trivial $R$-separable solutions.)

Put another way, a separable system is associated with a twodimensional subspace of commuting operators in $\mathscr{E}(3)^{2}$ and $S_{1}, S_{2}$ is a basis (non-unique) for the subspace. The group $E(3)$ acts on the set of all two-dimensional subspaces of commuting operators in $\mathscr{E}(3)^{2}$ via the adjoint representation $\left(S \rightarrow \boldsymbol{T}(g) S \boldsymbol{T}\left(g^{-1}\right), g \in E(3)\right)$, and decomposes this set into orbits of equivalent subspaces. One regards separable coordinates associated with equivalent subspaces as equivalent, since one can obtain any such system from any other by a Euclidean coordinates transformation. As proved in [4] there are eleven types of distinct orbits and they match exactly the eleven types of orthogonal separable coordinates. Representative operators from each orbit and the associated coordinate systems are listed in Table 1.

TABLE 1. Operators and separable coordinates for the Helmholtz equation.

\section{Commuting operators $S_{1}, S_{2}$}

\section{1. $P_{2}^{2}, P_{3}^{2}$}

2. $J_{3}^{2}, P_{3}^{2}$

3. $\left\{J_{3}, P_{2}\right\}, P_{3}^{2}$

4. $J_{3}^{2}+d^{2} P_{1}^{2}, P_{3}^{2}, d>0$

5. J.J, $J_{3}^{2}$

6. $J \cdot J-a^{2}\left(P_{1}^{2}+P_{2}^{2}\right)$, $J_{3}^{2}, a>0$

7. $J \cdot J+a^{2}\left(P_{1}^{2}+P_{2}^{2}\right)$, $J_{3}^{2}, a>0$

8. $\left\{J_{1}, P_{2}\right\}-\left\{J_{2}, P_{1}\right\}, J_{3}^{2}$

9. $J_{3}^{2}-c^{2} P_{3}^{2}+c\left(\left\{J_{2}, P_{1}\right\}\right.$ $\left.+\left\{J_{1}, P_{2}\right\}\right)$,

\section{Coordinates}

Cartesian $x, y, z$

Cylindrical

$x=r \cos \varphi, y=r \sin \varphi, z=z$

Parabolic cylindrical

$x=\frac{1}{2}\left(\xi^{2}-\eta^{2}\right), y=\xi \eta, z=z$

Elliptic cylindrical

$x=d \cos \alpha \cos \beta, y=d \sinh \alpha \sin \beta, z=z$

Spherical

$x=\rho \sin \theta \cos \varphi, y=\rho \sin \theta \sin \varphi, z=\rho \cos \theta$

Prolate spheroidal

$x=a \sinh \eta \sin \alpha \cos \varphi, y=a \sinh \eta \sin \alpha \sin \varphi$,

$z=a \cos \eta \cos \alpha$

Oblate spheroidal

$x=a \cosh \eta \sin \alpha \cos \varphi, y=a \cosh \eta \sin \alpha \sin \varphi$, $z=a \sinh \eta \cos \alpha$

Parabolic $x=\xi \eta \cos \varphi, y=\xi \eta \sin \varphi, z=\frac{1}{2}\left(\xi^{2}-\eta^{2}\right)$

Paraboloidal

$x=2 c \cosh \alpha \cos \beta \sinh \gamma$, 


$$
\begin{aligned}
c\left(P_{2}^{2}-P_{1}^{2}\right)+\left\{J_{2}, P_{1}\right\} & y=2 c \sinh \alpha \sin \beta \cosh \gamma, \\
-\left\{J_{1}, P_{2}\right\} & z=c(\cosh 2 \alpha+\cos 2 \beta-\cosh 2 \gamma) / 2
\end{aligned}
$$

10. $P_{1}^{2}+a P_{2}^{2}+(a+1) P_{3}^{2} \quad$ Ellipsoidal

$$
\begin{array}{rlrl}
\quad+J \cdot J, & x & =[((\mu-a)(\nu-a)(\rho-a)) /(a(a-1))]^{1 / 2} \\
J_{2}^{2}+a\left(J_{1}^{2}+P_{3}^{2}\right), & & y & =[((\mu-1)(\nu-1)(\rho-a)) /(1-a)]^{1 / 2} \\
a>1 & z & =[\mu \nu \rho / a]^{1 / 2}
\end{array}
$$

11. $\boldsymbol{J} \cdot \boldsymbol{J}, J_{1}^{2}+b J_{2}^{2}, \quad$ Conical

$$
\begin{array}{rl}
1>b>0 & x=r[((b \mu-1)(b \nu-1)) /(1-b)]^{1 / 2}, \\
y & =r[(b(\mu-1)(\nu-1)) /(b-1)]^{1 / 2}, \\
z & =r[b \mu \nu]^{1 / 2}
\end{array}
$$

Before embarking on a detailed study of these systems we present the analogous results for the Laplace equation (0.2). It is straightforward to check that, apart from the trivial symmetry $E$, the symmetry algebra of this equation is ten-dimensional. In addition to the six symmetries (1.2) which generate the subalgebra $\mathscr{E}(3)$ we have the generators

$$
\begin{aligned}
D & =-\left(\frac{1}{2}+x_{1} \partial_{1}+x_{2} \partial_{2}+x_{3} \partial_{3}\right) \\
K_{1} & =x_{1}+\left(x_{1}^{2}-x_{2}^{2}-x_{3}^{2}\right) \partial_{1}+2 x_{1} x_{3} \partial_{3}+2 x_{1} x_{2} \partial_{2} \\
K_{2} & =x_{2}+\left(x_{2}^{2}-x_{1}^{2}-x_{3}^{2}\right) \partial_{2}+2 x_{2} x_{3} \partial_{3}+2 x_{2} x_{1} \partial_{1} \\
K_{3} & =x_{3}+\left(x_{3}^{2}-x_{1}^{2}-x_{2}^{2}\right) \partial_{3}+2 x_{3} x_{1} \partial_{1}+2 x_{3} x_{2} \partial_{2} .
\end{aligned}
$$

Here $D$ is the generator of dilatations and the $K_{j}$ are generators of special conformal transformations to be discussed below. Only the elements of the $\mathscr{E}(3)$ subalgebra actually commute with the Laplace operator $\Delta_{3}$. The remaining elements of the Lie algebra merely leave the solution space of (0.2) invariant.

The real symmetry algebra generated by these elements is isomorphic to $s o(4,1)$, the Lie algebra of all real $5 \times 5$ matrices $\mathscr{A}$ such that $\mathscr{A} G^{4,1}$ $+G^{4,1} \mathscr{A}^{t}=0$ where

$$
G^{4,1}=\left(\begin{array}{lllll}
1 & & & & 0 \\
& 1 & & & \\
& & 1 & & \\
& & & 1 & \\
& & & & -1
\end{array}\right)=\sum_{j=1}^{4} \mathscr{E}_{j j}-\mathscr{E}_{55} .
$$

Here $\mathscr{E}_{i j}$ is the $5 \times 5$ matrix with the entry 1 in row $i$, column $j$, and 0 everywhere else. 


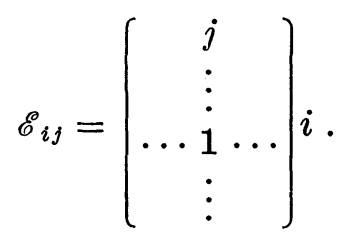

A basis for $s o(4,1)$ is provided by the ten elements

$$
\begin{aligned}
& \Gamma_{a b}=\mathscr{E}_{a b}-\mathscr{E}_{b a}=-\Gamma_{b a}, \quad 1 \leq a, b \leq 4 \\
& \Gamma_{a 5}=\mathscr{E}_{a 5}+\mathscr{E}_{5 a}=\Gamma_{5 a}
\end{aligned}
$$

where

$$
\begin{aligned}
{\left[\Gamma_{a b}, \Gamma_{c d}\right] } & =\delta_{b c} \Gamma_{a d}+\delta_{a d} \Gamma_{b c}+\delta_{c a} \Gamma_{a b}+\delta_{d b} \Gamma_{c a} \\
{\left[\Gamma_{a 5}, \Gamma_{c d}\right] } & =\delta_{a c} \Gamma_{a 5}-\delta_{a d} \Gamma_{c 5} \\
{\left[\Gamma_{a 5}, \Gamma_{b 5}\right] } & =\Gamma_{a d}
\end{aligned}
$$

One can verify that the correct commutation relations for the operators (1.2), (1.16) result if the following identifications are made:

$$
\begin{aligned}
& J_{1}=\Gamma_{43}, J_{2}=\Gamma_{24}, J_{3}=\Gamma_{32}, \quad D=\Gamma_{15} \\
& P_{1}=\Gamma_{12}+\Gamma_{25}, P_{2}=\Gamma_{13}+\Gamma_{35}, P_{3}=\Gamma_{14}+\Gamma_{45} \\
& K_{1}=\Gamma_{12}-\Gamma_{25}, K_{2}=\Gamma_{13}-\Gamma_{35}, K_{3}=\Gamma_{14}-\Gamma_{45} .
\end{aligned}
$$

The symmetry group of (0.2), the conformal group, is thus locally isomorphic to $S O(4,1)$, the group of all real $5 \times 5$ matrices $A$ such that

$$
A G^{4,1} A^{t}=G^{4,1} \text {. }
$$

The identity component of this group consists of those matrices satisfying (1.20), $\operatorname{det} A=1$ and $A_{55} \geq 1$. The Lie algebra of $S O(4,1)$ is $s o(4,1)$.

Exponentiating the operators (1.2),(1.16) we can obtain the local action of $S O(4,1)$ as a transformation group of symmetry operators. In particular, the momentum and angular momentum operators generate the subgroup of symmetries (1.12) isomorphic to $E(3)$, the dilatation operator generates

$$
\exp (\lambda D) \Psi(\boldsymbol{x})=e^{-\lambda / 2} \Psi\left(e^{-\lambda} \boldsymbol{x}\right), \quad \lambda \in \boldsymbol{R},
$$

and the $K_{j}$ generate the special conformal transformations

$$
\begin{aligned}
\exp (\boldsymbol{a} \cdot \boldsymbol{K}) \Psi(\boldsymbol{x})= & {[1-2 \boldsymbol{x} \cdot \boldsymbol{a}+(\boldsymbol{a} \cdot \boldsymbol{a}) \boldsymbol{x} \cdot \boldsymbol{x}]^{-1 / 2} } \\
& \cdot \Psi\left(\frac{\boldsymbol{x}-\boldsymbol{a}(\boldsymbol{x} \cdot \boldsymbol{x})}{1-2(\boldsymbol{a} \cdot \boldsymbol{x})(\boldsymbol{x} \cdot \boldsymbol{x})}\right) .
\end{aligned}
$$


In addition, we shall consider the inversion and space reflection symmetries of the Laplace equation:

$$
\begin{gathered}
I \Psi(\boldsymbol{x})=\frac{1}{\sqrt{\boldsymbol{x} \cdot \boldsymbol{x}}} \Psi(\boldsymbol{x} / \boldsymbol{x} \cdot \boldsymbol{x}), \\
R \Psi(\boldsymbol{x})=\Psi\left(-x_{1}, x_{2}, x_{3}\right), \\
I=I^{-1}, \quad R=R^{-1} .
\end{gathered}
$$

These are well-known symmetries of (0.2) which are not generated by the infinitesimal operators (1.2), (1.16), [7], page 31. It follows from the definitions of these operators that

$$
I P_{j} I^{-1}=-K_{j}, I D I^{-1}=-D, I J_{k} I^{-1}=J_{k} .
$$

The second-order symmetry operators of (0.2) can also be computed in a straightforward but tedious manner. One finds that modulo the trivial symmetries $\Phi \Delta_{3}, \Phi \in \mathscr{F}$, the space of truely second-order symmetries is fifteen-dimensional. It is obvious that any operator of the form $\left\{L_{1}, L_{2}\right\}, L_{j} \in s o(4,1)$, is a second-order symmetry and the space of such symmetric second-order elements in the enveloping algebra of so $(4,1)$ is thirty-five dimensional. However, there are twenty linearly independent relations between these operators which hold on the solution space of (0.2). Thus, only fifteen of these operators can be regarded as independent on the solution space. For example we have the relations

$$
\begin{aligned}
\text { i) } & \boldsymbol{P} \cdot \boldsymbol{P}=\boldsymbol{K} \cdot \boldsymbol{K}=\boldsymbol{P} \cdot \boldsymbol{J}=0 \\
\text { ii) } & \boldsymbol{J} \cdot \boldsymbol{J}=\frac{1}{4}-D^{2} \\
\text { iii) } & \Gamma_{45}^{2}+\Gamma_{41}^{2}-\Gamma_{51}^{2}=\frac{1}{4}+\Gamma_{23}^{2} \\
\text { iv) } & \left\{P_{1}, K_{1}\right\}+\left\{P_{2}, K_{2}\right\}+\left\{\boldsymbol{P}_{3}, K_{3}\right\}=2+4 D^{2},
\end{aligned}
$$

most of which are valid only when applied to solutions of (0.2), not in general.

We will see that all known $R$-separable orthogonal coordinate systems for (0.2) are characterized by a pair of commuting second-order symmetry operators in the enveloping algebra of $s o(4,1)$. Here two coordinate systems will be regarded as equivalent if one can be obtained from the other by a transformation from the connected component of the identity of the conformal group, augmented by the discrete symmetries (1.23). 


\section{Section 2. Separation of variables for the Helmholtz equation.}

We briefly study each of the eleven coordinate systems listed in Table 1 to determine the form of the separated solutions and the significance of the eigenvalues of the commuting symmetry operators. It is obvious that each of these systems also leads to variable separation in the Laplace equation (set $\omega=0$ ), so we can simultaneously present the results for this equation.

We begin by considering solutions $\Psi$ of the Helmholtz equation which are eigenfunctions of the operator $P_{3}$ :

$$
P_{3} \Psi=i \lambda \Psi, \quad \Psi(\boldsymbol{x})=e^{i \lambda z} \Phi(x, y)
$$

In this case we can separate the variable $z$ in (0.1) and reduce this equation to

$$
\left(\Delta_{2}+\omega^{2}-\lambda^{2}\right) \Phi(x, y)=0
$$

the Helmholtz equation in two variables. As is well-known, [3], equation (2.1) separates in four coordinate systems.

For Cartesian coordinates 1] the separated equations are

$$
\begin{aligned}
& X^{\prime \prime}+k^{2} X=0, Y^{\prime \prime}+\left(\omega^{2}-\lambda^{2}-k^{2}\right) Y=0 \\
& \Phi(x, y)=X(x) Y(y)=\exp (i k x) \exp \left( \pm i \sqrt{\omega^{2}-\lambda^{2}-k^{2}} y\right) .
\end{aligned}
$$

The corresponding separated solutions of $(0.1)$ are characterized by the equations

$$
P_{3} \Psi=i \lambda \Psi, \quad P_{1} \Psi=i k \Psi
$$

The associated Laplace equation solutions are obtained by setting $\omega=0$ in (2.1), (2.2).

For cylindrical coordinates 2] the separated equations are, $(\Phi=\Theta R)$ :

$$
\Theta^{\prime \prime}+m^{2} \Theta=0, \quad r^{2} R^{\prime \prime}+r R^{\prime}+\left[r^{2}\left(\omega^{2}-\lambda\right)-m^{2}\right] R=0
$$

with solutions

$$
\Theta(\theta)=e^{i m \theta}, \quad R(r)=J_{ \pm m}\left(\sqrt{\omega^{2}-\lambda^{2}} r\right)
$$

where $J_{\nu}(z)$ is a Bessel function. Here, the separated solutions $\Psi$ are characterized by the equations

$$
P_{3} \Psi=i \lambda \Psi, \quad i J_{3} \Psi=m \Psi .
$$


For the Laplace equation $\left(\omega^{2}=0\right)$ the results are the same.

For parabolic cylindrical coordinates 3] the separated equations are

$$
\begin{aligned}
& C^{\prime \prime}+\left[-k^{2}+\left(\omega^{2}-\lambda^{2}\right) \xi^{2}\right] C=0 \\
& N^{\prime \prime}+\left[k^{2}+\left(\omega^{2}-\lambda^{2}\right) \eta^{2}\right] N=0 \\
& \Phi=C(\xi) N(\eta)=D_{i \mu-1 / 2}( \pm \sigma \xi) D_{-i \mu-1 / 2}( \pm \sigma \eta) \\
& \sigma=e^{i \pi / 4} \sqrt{2}\left(\omega^{2}-\lambda^{2}\right)^{1 / 4}, \quad k^{2}=2 \mu \sqrt{\omega^{2}-\lambda^{2}}
\end{aligned}
$$

Here $D_{\nu}(z)$ is a parabolic cylinder function. The separated solutions are characterized by the eigenvalue equations

$$
P_{3} \Psi=i \lambda \Psi, \quad\left\{J_{3}, P_{2}\right\} \Psi=k^{2} \Psi
$$

The corresponding results for the Laplace equation are obtained by setting $\omega=0$ in (2.6).

For elliptic cylinder coordinates the separated equations are

$$
\begin{aligned}
& A^{\prime \prime}+\left[d^{2}\left(\omega^{2}-\lambda^{2}\right) \cosh ^{2} \alpha+k\right] A=0 \\
& B^{\prime \prime}-\left[d^{2}\left(\omega^{2}-\lambda^{2}\right) \cos ^{2} \beta+k\right] B=0 \\
& \Phi=A(\alpha) B(\beta)= \begin{cases}C e_{n}(\alpha, q) c e_{n}(\beta, q), & n=0,1, \ldots \\
\operatorname{Se}_{n}(\alpha, q) \operatorname{se}_{n}(\beta, q), & n=1,2, \cdots\end{cases} \\
& q=d^{2}\left(\omega^{2}-\lambda^{2}\right) / 4 .
\end{aligned}
$$

The separated equations are forms of Mathieu's equation. For $\lambda$ real, $\omega>|\lambda|$ one obtains single-valued solutions in all of three-space if and only if $k$ is one of a discrete set of eigenvalues $k_{n}$. The corresponding solutions are expressed in terms of Mathieu functions $c e_{n}(\beta), s e_{n}(\beta)$ and associated Mathieu functions $C e_{n}(\alpha), S e_{n}(\alpha)$, see [10]. For other values of $\lambda$ similar comments hold. The eigenvalue equations are

$$
P_{3} \Psi=i \lambda \Psi, \quad\left(J_{3}^{2}+d^{2} P_{1}^{2}\right) \Psi=k \Psi .
$$

Again the results for the Laplace equation follow essentially from (2.8) by setting $\omega=0$.

Next we consider solutions $\Psi$ of (0.1) which are eigenfunctions of $J_{3}$ :

$$
i J_{3}=m \Psi, \quad \Psi(x, y, z)=e^{i m \varphi} \Phi(r, z) .
$$

Here $r, \varphi, z$ are cylindrical coordinates 2] and $J_{3}=-\partial_{\varphi}$. We now split off the variable $\varphi$ so that $(0.1)$ reduces to 


$$
\left(\partial_{r r}+\frac{1}{r} \partial_{r}-\frac{m^{2}}{r^{2}}+\partial_{z z}+\omega^{2}\right) \Phi=0
$$

This reduced equation separates in five coordinate systems corresponding to systems 2], 5], 6], 7], 8] on Table 1.

For spherical coordinates 5] the separated equations in $\rho, \theta$ are

$$
\begin{aligned}
& \text { a) } P^{\prime \prime}+\frac{2}{\rho} P^{\prime}+\left(\omega^{2}-\frac{\ell(\ell+1)}{\rho^{2}}\right) P=0 \\
& \text { b) } \Theta^{\prime \prime}+\cot \theta \Theta^{\prime}+\left(\ell(\ell+1)-\frac{m^{2}}{\sin ^{2} \theta}\right) \Theta=0 \\
& \Phi=P(\rho) \Theta(\theta)=\rho^{-1 / 2} J_{ \pm(\ell+1 / 2)}(\omega \rho) P_{\ell}^{ \pm m}(\cos \theta)
\end{aligned}
$$

Here $J_{\nu}(z)$ is a Bessel function and $P_{\ell}^{m}(\cos \theta)$ is an associated Legendre function, [11]. The eigenvalue equations characterizing the spherical system are

$$
i J_{3} \Psi=m \Psi, \quad J \cdot J \Psi=-\ell(\ell+1) \Psi .
$$

The separated equations for the Laplace equation are obtained from (2.11) by setting $\omega=0$. The $\Theta$ solution is unchanged but a basis for a) becomes $P(\rho)=\rho^{\ell}, \rho^{-\ell-1}$.

For prolate spheroidal coordinates 6] the separated equations in $\eta$, $\alpha$ are

$$
\begin{aligned}
& H^{\prime \prime}+\operatorname{coth} \eta H^{\prime}+\left(-\lambda+a^{2} \omega^{2} \sinh ^{2} \eta-\frac{m^{2}}{\sinh ^{2} \eta}\right) H=0 \\
& A^{\prime \prime}+\cot \alpha A^{\prime}+\left(\lambda+a^{2} \omega^{2} \sin ^{2} \alpha-\frac{m^{2}}{\sin ^{2} \alpha}\right) A=0 \\
& \left(J \cdot J-a^{2}\left(P_{1}^{2}+P_{2}^{2}\right)\right) \Psi=-\lambda \Psi, \quad i J_{3} \Psi=m \Psi .
\end{aligned}
$$

Equations (2.13) are two forms of the spheroidal wave equation, [10]. The corresponding solutions of (1.1) which are bounded and single valued in $\boldsymbol{R}^{3}$ are of the form

$$
\begin{aligned}
\Psi=H(\eta) A(\alpha) e^{i m \varphi}=S_{n}^{m(1)}(\cosh \eta, a \omega) P s_{n}^{|m|}\left(\cos \alpha, a^{2} \omega^{2}\right) e^{i m \varphi} \\
\\
n=0,1,2, \cdots, m=n, n-1, \cdots,-n,
\end{aligned}
$$

where $S_{n}^{m(1)}, P s_{n}^{m}(z, \gamma)$ are spheroidal wave functions. The discrete eigenvalues $\lambda_{n}^{|m|}\left(a^{2} \omega^{2}\right)$ are analytic functions of $a^{2} \omega^{2}$ and $\lambda_{n}^{|m|}(0)=n(n+1)$. The coordinates vary in the range $0 \leq \alpha<2 \pi, 0 \leq \eta, 0 \leq \varphi<2 \pi$. For the Laplace equation the separated equations are (2.13) with $\omega=0$ and the separated 
solutions take the form

$$
\Psi=P_{n}^{ \pm m}(\cosh \eta) P_{n}^{ \pm m}(\cos \alpha) e^{i m \varphi} .
$$

For oblate spheroidal coordinates 7] the separated equations in $\eta, \alpha$ are

$$
\begin{aligned}
& H^{\prime \prime}+\tanh \eta H^{\prime}+\left(-\lambda+a^{2} \omega^{2} \cosh ^{2} \eta+\frac{m^{2}}{\cosh ^{2} \eta}\right) H=0 \\
& A^{\prime \prime}+\cot \alpha A^{\prime}+\left(\lambda-a^{2} \omega^{2} \sin ^{2} \alpha-\frac{m^{2}}{\sin ^{2} \alpha}\right) A=0 \\
& \left(\boldsymbol{J} \cdot \boldsymbol{J}+a^{2}\left(P_{1}^{2}+P_{2}^{2}\right)\right) \Psi=-\lambda \Psi, \quad i J_{3} \Psi=m \Psi .
\end{aligned}
$$

Again these equations are forms of the spheroidal wave equation. The corresponding solutions $\Psi$ which are bounded and single-valued in $\boldsymbol{R}^{3}$ take the form

$$
S_{n}^{m(1)}(-i \sinh \eta, i a \omega) P s_{n}^{|m|}\left(\cos \alpha,-a^{2} \omega^{2}\right) e^{i m \varphi}
$$

with eigenvalues $\lambda_{n}^{|m|}\left(-a^{2} \omega^{2}\right)$. For the Laplace equation the eigenfunctions are of the form

$$
P_{n}^{ \pm m}(-i \sinh \eta) P_{n}^{ \pm m}(\cos \alpha) e^{i m \varphi} .
$$

For parabolic coordinates 8 ] the separated equations in $\xi, \eta$ are

$$
\begin{aligned}
& \Xi^{\prime \prime}+\frac{1}{\xi} \Xi^{\prime}+\left(\omega^{2} \xi^{2}-\frac{m^{2}}{\xi^{2}}+\lambda\right) \Xi=0 \\
& H^{\prime \prime}+\frac{1}{\eta} H^{\prime}+\left(\omega^{2} \eta^{2}-\frac{m^{2}}{\eta^{2}}-\lambda\right) H=0 \\
& \left(\left\{J_{1}, P_{2}\right\}-\left\{J_{2}, P_{1}\right\}\right) \Psi=\lambda \Psi, \quad i J_{3} \Psi=m \Psi,
\end{aligned}
$$

and the separated solutions $\Psi=\Xi(\xi) H(\eta) e^{i m \varphi}$ take the form

$$
\begin{aligned}
& \Xi(\xi)=\xi^{m} e^{ \pm i \omega \xi 2 / 2}{ }_{1} F_{1}\left(\begin{array}{c}
\frac{-i \lambda}{4 \omega}+\frac{m+1}{2} \\
m+1
\end{array} \mid \mp i \omega \xi^{2}\right), \\
& H(\eta)=\eta^{m} e^{ \pm i \omega \eta 2 / 2} F_{1}\left(\begin{array}{c}
\frac{i \lambda}{4 \omega}+\frac{m+1}{2} \\
m+1
\end{array} \mid \mp i \omega \eta^{2}\right) .
\end{aligned}
$$

In the case $\omega=0$ (Laplace equation) the separated solutions are

$$
J_{ \pm}(\sqrt{\lambda \xi}) J_{ \pm m}(i \sqrt{\lambda} \eta) e^{i m \varphi} .
$$

The above eight systems are the only ones for the Helmholtz equa- 
tion whose separated solutions are eigenfunctions of a second-order operator which is the square of a first-order symmetry operator. The remaining three systems are somewhat less tractible.

For paraboloidal coordinates 9] the separated equations in $\alpha, \beta, \gamma$ are

$$
\begin{gathered}
A^{\prime \prime}+\left(-q-\lambda c \cosh 2 \alpha+\frac{\omega^{2} c^{2}}{2} \cosh 4 \alpha\right) A=0 \\
B^{\prime \prime}+\left(q+\lambda c \cos 2 \beta-\frac{\omega^{2} c^{2}}{2} \cos 4 \beta\right) B=0 \\
\Gamma^{\prime \prime}+\left(-q+\lambda c \cosh 2 \lambda+\frac{\omega^{2} c^{2}}{2} \cosh 4 \gamma\right) \Gamma=0, \quad q=\mu-\frac{c^{2} \omega^{2}}{2}
\end{gathered}
$$

where

$$
\begin{aligned}
& \left(J_{3}^{2}-c^{2} P_{3}^{2}+c\left\{J_{2}, P_{1}\right\}+c\left\{J_{1}, P_{2}\right\}\right) \Psi=-\mu \Psi, \\
& \left(c P_{2}^{2}-c P_{1}^{2}+\left\{J_{2}, P_{1}\right\}-\left\{J_{1}, P_{2}\right\}\right) \Psi=\lambda \Psi .
\end{aligned}
$$

Each of the equations (2.22) can be transformed to the Whittaker-Hill equation

$$
\frac{d^{2} g}{d \theta^{2}}+\left(\mu+\frac{\omega^{2}}{8}+\omega \rho \cos 2 \theta-\frac{\omega^{2}}{8} \cos 4 \theta\right) g=0
$$

whose even and odd solutions which are periodic with period $2 \pi$ in $\theta$ are denoted $g c_{n}(\theta, \omega, \rho), g s_{n}(\theta, \omega, \rho)$, respectively. The subscript $n$ (the number of zeros in the interval $[0,2 \pi]$ ) labels the discrete eigenvalues $\mu=\mu_{n}$. See [12] for a complete discussion. Single-valued separable solutions $\Psi$ of (0.1) take the form

$$
\begin{aligned}
\Psi= & A B \Gamma=g c_{n}(i \alpha, 2 c \omega, \lambda / 2 \omega) g c_{n}(\beta, 2 c \omega, \lambda / 2 \omega) \\
& \times g c_{n}(i \gamma+\pi / 2,2 c \omega, \lambda / 2 \omega), \quad n=0,1,2, \cdots
\end{aligned}
$$

or the same form with $g c_{n}$ replaced by $g s_{n}$. Corresponding to the Laplace equation $(\omega=0)$ the separated solutions are Mathieu functions of the form

$$
\begin{aligned}
& C e_{n}(\alpha,-\lambda c / 2) c e_{n}(\beta,-\lambda c / 2) C e_{n}(\gamma+i \pi / 2,-\lambda c / 2), \\
& S e_{n}(\alpha,-\lambda c / 2) s e_{n}(\beta,-\lambda c / 2) S e_{n}(\gamma+i \pi / 2,-\lambda c / 2) .
\end{aligned}
$$

For ellipsoidal coordinates 10] where $0<\rho<1<\nu<a<\mu<\infty$ for single-valued coordinates, the separation equations all take the form

$$
\left(4 \sqrt{h(\xi)} \frac{d}{d \xi} \sqrt{h(\xi)} \frac{d}{d \xi}+\lambda_{1} \xi+\lambda_{2} \omega^{2} \xi^{2}\right) E(\xi)=0
$$




$$
h(\xi)=(\xi-a)(\xi-1) \xi, \quad \xi=\mu, \nu, \rho,
$$

where

$$
\begin{aligned}
& \left(J \cdot J+P_{1}^{2}+a P_{2}^{2}+(a+1) P_{3}^{2}\right) \Psi=\lambda_{1} \Psi, \\
& \left(J_{2}^{2}+a J_{1}^{2}+a P_{3}^{2}\right) \Psi=\lambda_{2} \Psi .
\end{aligned}
$$

For computational purposes it is more convenient to introduce equivalent separable coordinates $\alpha, \beta, \gamma$ defined by

$$
\rho=s n^{2}(\alpha, k), \quad \nu=s n^{2}(\beta, k), \quad \mu=\operatorname{sn}^{2}(\gamma, k), \quad k=a^{-1 / 2}
$$

where $\operatorname{sn}(z, k)$ is a Jacobi elliptic function, [10]. The relationship between $\alpha, \beta, \gamma$ and $x, y, z$ is

$$
\begin{aligned}
& x=\frac{i}{k k^{\prime}} d n \alpha d n \beta d n \gamma, \quad y=-\frac{k}{k^{\prime}} \operatorname{cn} \alpha \operatorname{cn} \beta c n \gamma, \\
& z=k \operatorname{sn} \alpha \operatorname{sn} \beta \operatorname{sn} \gamma
\end{aligned}
$$

where $c n \alpha, d n \alpha$ are elliptic functions and $k^{\prime}=\sqrt{1-k^{2}}$. To obtain real values for $x, y, z$ we choose $\alpha$ real, $\beta$ complex such that $\operatorname{Re} \beta=K$ and $\gamma$ complex such that $\operatorname{Im} \gamma=K^{\prime}$ where $K(k)$ is the modulus of elliptic functions and $K^{\prime}=K\left(k^{\prime}\right)$. To cover all real values of $x, y, z$ once it is sufficient to allow $\alpha \in[-K, K], \beta \in\left[K-i K^{\prime}, K+i K^{\prime}\right]$, parallel to the imaginary axis, and $\gamma \in\left[-K+i K^{\prime}, K+i K^{\prime}\right]$, parallel to the real axis. In the new variables the separation equations become the ellipsoidal wave equation

$$
\left\{\frac{d^{2}}{d \xi^{2}}+k^{2} \lambda_{2}+k^{2} \lambda_{1} s n^{2} \xi+k^{2} \omega^{2} s n^{4} \xi\right\} E(\xi)=0, \quad \xi=\alpha, \beta, \gamma,
$$

see $[10]$.

From the periodicity properties of elliptic functions it follows that if $\xi$ is replaced by $\xi+4 K n+4 i K^{\prime} m$ in (2.28) where $m$ and $n$ are integers and $\xi$ is any one of $\alpha, \beta, \gamma$, then $x, y, z$ remain unchanged. Thus only those solutions $E(\xi)$ of (2.29) which are double-periodic and single-valued in $\xi$ with real period $4 K$ and imaginary period $4 i K^{\prime}$ are single-valued functions of $x, y, z$. These solutions are called ellipsoidal wave functions

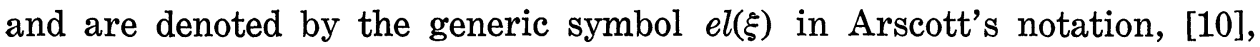
Chapter X. There are eight types of such functions, each expressable in the form

$$
s n^{r} z c n^{s} z d n^{t} z F\left(s n^{2} z\right), \quad r, s, t=0,1
$$


where $F$ is a convergent power series in its argument. The eigenvalues $\lambda_{1}, \lambda_{2}$ are countable and discrete.

Corresponding to the Laplace equation $(\omega=0)$, (2.29) reduces to the Lamé equation and the single-valued solutions in $\boldsymbol{R}^{3}$ are products of three Lamé polynomials, see [10], page 228.

For conical coordinates $r, \mu, \nu, 11]$ it is convenient to set $\mu=s n^{2}(\alpha, k)$, $v=s n^{2}(\beta, k)$, where $k=b^{-1 / 2}>0$. Then

$$
x=\frac{r}{k^{\prime}} d n \alpha d n \beta, \quad y=\frac{i r k}{k^{\prime}} \operatorname{cn} \alpha \operatorname{cn} \beta, \quad z=r k \operatorname{sn} \alpha \operatorname{sn} \beta
$$

and the variables have the range $0 \leq r, \alpha \in[-2 K, 2 K], \beta \in\left[K, K+2 i K^{\prime}\right]$, see [10], page 24. The separation equations are

$$
\begin{aligned}
& R^{\prime \prime}+\frac{2}{r} R^{\prime}+\left(\omega^{2}-\frac{\ell(\ell+1)}{r^{2}}\right) R=0 \\
& A^{\prime \prime}+\left(\lambda-\ell(\ell+1) k^{2} s n^{2} \alpha\right) A=0 \\
& B^{\prime \prime}+\left(\lambda-\ell(\ell+1) k^{2} s n^{2} \beta\right) B=0 \\
& J \cdot J \Psi=-\ell(\ell+1) \Psi, \quad\left(J_{1}^{2}+b J_{2}^{2}\right) \Psi=\lambda \Psi .
\end{aligned}
$$

The first equation has solutions of the form $R(r)=r^{-1 / 2} J_{ \pm(\ell+1 / 2)}(\omega r)$. The next two equations are examples of the Lamé equation. If $\alpha$ or $\beta$ is increased by integral multiples of $4 K$ or $4 i K^{\prime}$ it follows from (2.30) that $x, y$ and $z$ are unchanged. Thus only those solutions $A(\alpha), B(\beta)$ of (2.31) which are doubly-periodic and single-valued in $\alpha, \beta$ lead to single-valued functions of $x, y, z$. It is known that double-periodic solutions of Lamé's equation exist only in the cases $\ell=0,1,2, \ldots$, [10]. Furthermore, for such $\ell$ there exist exactly $2 \ell+1$ solutions corresponding to $2 \ell+1$ distinct eigenvalues $\lambda$. The solutions, one for each pair of eigenvalues $\lambda, \ell$ can be expressed as finite series called Lamé polynomials. There are eight types of these polynomials, each expressible in the form

$$
\begin{aligned}
& s n^{a} \alpha c n^{b} \alpha d n^{c} \alpha F_{P}\left(s n^{2} \alpha\right), \quad a, b, c=0,1, \\
& a+b+c+2 P=\ell
\end{aligned}
$$

where $F_{P}(z)$ is a polynomial of order $P$ in $z$.

Corresponding to the Laplace equation $(\omega=0)$ the above discussion is unchanged except that a basis of solutions for the first equation (2.31) becomes $r^{\ell}, r^{-\ell-1}$. In this case the solutions $\Psi$ are called ellipsoidal harmonics. 


\section{Section 3. A Hilbert space model.}

In analogy with the methods discussed in [2] we can introduce a Hilbert space structure on the solution space of (0.1) in such a way that the separated solutions can be interpreted as eigenfunctions of self-adjoint operators in the enveloping algebra of $\mathscr{E}(3)$. We proceed by expanding solutions of the Helmholtz equation in plane waves. One can easily show that $\Psi(\boldsymbol{x})$ is a solution of $(0.1)$ if it can be represented in the form

$$
\Psi(\boldsymbol{x})=\int_{S_{2}} \exp (i \omega \boldsymbol{x} \cdot \boldsymbol{k}) h(\boldsymbol{k}) d \Omega(\boldsymbol{k})=I(h), \quad \boldsymbol{k}=\left(k_{1}, k_{2}, k_{3}\right) .
$$

Here $\boldsymbol{k}$ is a unit vector $(\boldsymbol{k} \cdot \boldsymbol{k}=1)$ which runs over the unit sphere $S_{2}: k_{1}^{2}+k_{2}^{2}+k_{3}^{2}=1, d \Omega$ is the usual solid angle measure on the sphere and $h$ is a complex-valued measurable function on $S_{2}$ (with respect to $d \Omega$ ) such that

$$
\int_{S_{2}}|h|^{2} d \Omega<\infty
$$

The set $L_{2}\left(S_{2}\right)$ of such functions $h$ is a Hilbert space with inner product

$$
\left\langle h_{1}, h_{2}\right\rangle=\int_{S_{2}} h_{1}(\boldsymbol{k}) \overline{h_{2}}(\boldsymbol{k}) d \Omega(\boldsymbol{k}),
$$

where in terms of spherical coordinates on $S_{2}$

$$
\begin{gathered}
\boldsymbol{k}=(\sin \theta \cos \varphi, \sin \theta \sin \varphi, \cos \theta) \\
0 \leq \theta \leq \pi, \quad-\pi \leq \varphi<\pi, \quad d \Omega(\boldsymbol{k})=\sin \theta d \theta d \varphi .
\end{gathered}
$$

The elements $g(A, a)$ of $E(3)$ act on solutions of (0.1) via the operators $T(g)$, (1.9), (1.12). Using (3.1) we find

$$
\Psi=I(h) \Rightarrow \boldsymbol{T}(g) \Psi=I(T(g) h)
$$

where the operators $T(g)$ on $L_{2}\left(S_{2}\right)$ are defined by

$$
\begin{aligned}
\boldsymbol{T}(g) h(\boldsymbol{k}) & =\exp [i \omega \boldsymbol{a} \cdot(\boldsymbol{k} A)] h(\boldsymbol{k} A) \\
g & =(A, \boldsymbol{a}), \quad A \in S O(3), \boldsymbol{a} \in \boldsymbol{R}^{3} .
\end{aligned}
$$

Thus the $\boldsymbol{T}(g)$ acting on $\Psi$ induce operators (which we also call $\boldsymbol{T}(g)$ ) acting on $h$. Moreover, it is well-known that the $\boldsymbol{T}(g)$ on $L_{2}\left(S_{2}\right)$ define a unitary irreducible representation of $E(3)$, [8].

The Lie algebra representation of $\mathscr{E}(3)$ on $L_{2}\left(S_{2}\right)$ induced by the generators (1.2) on the solution space is determined by 


$$
\begin{aligned}
& P_{1}=i \omega k_{1}=i \omega \sin \theta \cos \varphi, \quad P_{2}=i \omega k_{2}=i \omega \sin \theta \sin \varphi \\
& P_{3}=i \omega k_{3}=i \omega \cos \theta, \quad J_{3}=k_{2} \partial_{1}-k_{1} \partial_{2}=-\partial_{\varphi} \\
& J_{1}=k_{3} \partial_{2}-k_{2} \partial_{3}=\sin \varphi \partial_{\theta}+\cos \varphi \cot \theta \partial_{\varphi} \\
& J_{2}=k_{1} \partial_{3}-k_{3} \partial_{1}=-\cos \varphi \partial_{\varphi}+\sin \varphi \cot \theta \partial_{\varphi} .
\end{aligned}
$$

These Lie algebra operators are related to the operators $T(g)$, (3.5), by (1.12). Furthermore, the operators (3.6) are skew-Hermitian on the dense subspace $\mathscr{D}$ of $L_{2}\left(S_{2}\right)$ consisting of $C^{\infty}$ functions on $S_{2}$. The corresponding elements of $\mathscr{E}(3)^{2}$ are easily seen to be symmetric on $\mathscr{D}$ and we shall show explicitly that their domains can be extended to define self-adjoint operators on dense subspaces of $L_{2}\left(S_{2}\right)$. Corresponding to each pair of commuting operators listed in Table 1 we shall find a pair of commuting self-adjoint operators $S, S^{\prime}$ on $L_{2}\left(S_{2}\right)$ and determine the spectral resolution of this pair. These results can then be used to obtain information about the space $\mathscr{H}=I\left(L_{2}\left(S_{2}\right)\right)$. Here $\mathscr{H}$ is a Hilbert space with inner product

$$
\left(\Psi_{1}, \Psi_{2}\right) \equiv\left\langle h_{1}, h_{2}\right\rangle, \quad \Psi_{j}=I\left(h_{j}\right)
$$

(It is not hard to show that no nonzero $h \in L_{2}\left(S_{2}\right)$ can be mapped by $I$ to the zero solution of (0.1).) It follows that $I$ is a unitary transformation from $L_{2}\left(S_{2}\right)$ to $\mathscr{H}$ and the operators $T(g),(1.9),(1.12)$, on $\mathscr{H}$ are unitary.

We can also interpret each $\Psi \in \mathscr{H}$ as an inner product

$$
\begin{aligned}
\Psi(\boldsymbol{x}) & =I(h)=\langle h, H(\boldsymbol{x}, \cdot)\rangle \\
H(\boldsymbol{x}, \boldsymbol{k}) & =\exp [-i \omega \boldsymbol{x} \cdot \boldsymbol{k}] \in L_{2}\left(S_{2}\right),
\end{aligned}
$$

see [13].

The existence of the unitary mapping $I$ allows us to transform problems involving $\mathscr{H}$ to problems involving $L_{2}\left(S_{2}\right)$. In particular, if $S$, $S^{\prime}$ are a pair of commuting operators from Table 1 we can interpret them as a pair of commuting self-adjoint operators on $L_{2}\left(S_{2}\right)$ and compute a basis for $L_{2}\left(S_{2}\right)$ consisting of (generalised) eigenfunctions

$$
S f_{\lambda \mu}=\lambda f_{\lambda \mu}, \quad S^{\prime} f_{\lambda \mu}=\mu f_{\lambda \mu}, \quad\left\langle f_{\lambda \mu}, f_{\lambda^{\prime} \mu^{\prime}}\right\rangle=\delta\left(\lambda-\lambda^{\prime}\right) \delta\left(\mu-\mu^{\prime}\right) .
$$

Then the functions $\Psi_{\lambda \mu}(x)=I\left(f_{\lambda \mu}\right)$ will form a corresponding basis in $\mathscr{H}$ for the operators $S, S^{\prime}$ constructed from the generators (1.2):

$$
S \Psi_{\lambda \mu}=\lambda \Psi_{\lambda \mu}, \quad S^{\prime} \Psi_{\lambda \mu}=\mu \Psi_{\lambda \mu} .
$$


These last expressions enable us to evaluate the integral $I\left(f_{\lambda \mu}\right)$, for they guarantee that $\Psi_{\lambda \mu}$ is solution of $(0.1)$ which is separable in the coordinates associated with $S, S^{\prime}$. Furthermore, if $\Psi=I(h)$ for some $h \in L_{2}\left(S_{2}\right)$ we have the expansion

$$
\boldsymbol{T}(g) \Psi(\boldsymbol{x})=\sum_{\lambda, \mu}\left\langle\boldsymbol{T}(g) h, f_{\lambda \mu}\right\rangle \Psi_{\lambda \mu}(\boldsymbol{x})
$$

which converges both pointwise and in the Hilbert space sense.

Since the spherical coordinate system 5] is treated in detail in many textbooks, e.g., [8], [9], we shall here list only the most important facts concerning this system, omitting all proofs.

The unitary irreducible representations of $S O(3)$ are denoted $D_{\ell}, \ell$ $=0,1,2, \cdots$ where $\operatorname{dim} D_{\ell}=2 \ell+1$. If $\left\{J_{1}, J_{2}, J_{3}\right\}$ are the operators on the representation space $V_{\ell}$ of $D_{\ell}$ which correspond to the Lie algebra generators (1.5) then there is an orthonormal basis $\left\{f_{m}^{(\ell)}: m=\ell, \ell-1\right.$, $\cdots,-\ell\}$ for $V_{\ell}$ such that

$$
\begin{gathered}
J^{0} f_{m}^{(\ell)}=m f_{m}^{(\ell)}, \quad J^{ \pm} f_{m}^{(\ell)}=[(\ell \pm m+1)(\ell \mp m)]^{1 / 2} f_{m \pm 1}^{(\ell)} \\
J^{ \pm}=\mp J_{2}+i J_{1}, \quad J^{0}=i J_{3} .
\end{gathered}
$$

The matrix elements of the group operators $T(A), A \in S O(3)$, with respect to this basis are the Wigner $D$-functions, [8], [9]. It follows from (3.12) that on $V_{\ell}, \boldsymbol{J} \cdot \boldsymbol{J}=-\ell(\ell+1)$.

Now consider the representation $T$ of $E(3)$ on $L_{2}\left(S_{2}\right)$ defined by (3.5). The restriction of $T$ to the subgroup $S O(3)$ breaks up into the direct sum

$$
T \mid S O(3) \cong \sum_{\ell=0}^{\infty} \oplus D_{\ell}, \quad L_{2}\left(S_{2}\right) \cong \sum_{\ell=0}^{\infty} V_{\ell}
$$

where $\operatorname{dim} V_{\ell}=2 \ell+1$ and the action of the operators $T(A)$ on the invariant subspace $V_{\ell}$ is equivalent to $D_{\ell}$. The elements $h$ of $V_{\ell}$ are characterized as the solutions of $\boldsymbol{J} \cdot \boldsymbol{J} h=-\ell(\ell+1) h$ or

$$
\left(\partial_{\theta \theta}+\cot \theta \partial_{\theta}+\frac{1}{\sin ^{2} \theta} \partial_{\varphi \varphi}\right) h(\theta, \varphi)=-\ell(\ell+1) h(\theta, \varphi)
$$

in terms of coordinates (3.3). Here, $\boldsymbol{J} \cdot \boldsymbol{J}$ is known as the Laplace operator on the sphere $S_{2}$. It follows from these facts that the self-adjoint extension of this operator (which we also call $\boldsymbol{J} \cdot \boldsymbol{J}$ ) has discrete spectrum $-\ell(\ell+1), \quad \ell=0,1,2, \cdots$, each eigenvalue occuring with multiplicity $2 \ell+1$. 
There exists a basis for $V_{\ell}$ consisting of eigenfunctions $f_{m}^{(\ell)}(\theta, \varphi)$ of the symmetry operator $J^{0}$, which satisfy relations (3.12) where

$$
J^{ \pm}=e^{ \pm i \varphi}\left( \pm \partial_{\theta}+i \cot \theta \partial_{\varphi}\right), \quad J^{0}=-i \partial_{\varphi} .
$$

Indeed from the recurrence relations (3.12) and the differential equation (3.13) one finds

$$
f_{m}^{(\ell)}(\theta, \varphi)=Y_{\ell}^{m}(\theta, \varphi), \quad\left\langle Y_{\ell}^{m}, Y_{\ell^{\prime}}^{m^{\prime}}\right\rangle=\delta_{\ell \ell^{\prime}} \delta_{m m^{\prime}}
$$

where

$$
Y_{\ell}^{m}(\theta, \varphi)=\left[\frac{(2 \ell+1)(\ell-m) !}{4 \pi(\ell+m) !}\right]^{1 / 2} P_{\ell}^{m}(\cos \theta) e^{i m \varphi}
$$

is a spherical harmonic.

Furthermore, it is straightforward to show that the action of the operators $P_{j}$ on the basis is

$$
\begin{aligned}
P^{0} f_{m}^{(\ell)}= & -\omega\left[\frac{(\ell+m+1)(\ell-m+1)}{(2 \ell+3)(2 \ell+1)}\right]^{1 / 2} f_{m}^{(\ell+1)} \\
& -\omega\left[\frac{(\ell+m)(\ell-m)}{(2 \ell+1)(2 \ell-1)}\right]^{1 / 2} f_{m}^{(\ell-1)} \\
P^{+} f_{m}^{(\ell)}=\omega\left[\frac{(\ell+m+1)(\ell+m+2)}{(2 \ell+3)(2 \ell+1)}\right]^{1 / 2} f_{m+1}^{(\ell+1)} & -\omega\left[\frac{(\ell-m)(\ell-m-1)}{(2 \ell+1)(2 \ell-1)}\right]^{1 / 2} f_{m+1}^{(\ell-1)} \\
P^{-} f_{m}^{(\ell)}= & -\omega\left[\frac{(\ell-m+2)(\ell-m+1)}{(2 \ell+3)(2 \ell+1)}\right]^{1 / 2} f_{m-1}^{(\ell+1)} \\
& +\omega\left[\frac{(\ell+m)(\ell+m-1)}{(2 \ell+1)(2 \ell-1)}\right]^{1 / 2} f_{m-1}^{(\ell-1)}
\end{aligned}
$$

where

$$
P^{0}=i P_{3}=-\omega \cos \theta, \quad P^{ \pm}=\mp P_{2}+i P_{1}=-\omega e^{ \pm i \varphi} \sin \theta ，
$$

e.g., [14].

Applying the integral transformation $I$ to our basis $\left\{f_{m}^{(\ell)}\right\}$ we obtain an orthonormal basis $\left\{\Psi_{m}^{(\ell)}=I\left(f_{m}^{(\ell)}\right)\right\}$ for $\mathscr{H}$ which satisfies the eigenvalue equations

$$
J \cdot J \Psi_{m}^{(\ell)}=-\ell(\ell+1) \Psi_{m}^{(\ell)}, \quad i J_{3} \Psi_{m}^{(\ell)}=m \Psi_{m}^{(\ell)}
$$

These eigenfunctions separate in the spherical coordinate system 5] and 
are explicitly given by

$$
\Psi_{m}^{(\ell)}(\rho, \theta, \varphi)=\left(\frac{8 \pi^{3}}{\omega \rho}\right)^{1 / 2} i^{\ell} J_{\ell+1 / 2}(\omega \rho) Y_{\ell}^{m}(\theta, \varphi) .
$$

These (standing) spherical waves necessarily satisfy the recurrence relations (3.12), (3.17) where now the operators $J_{j}, P_{j}$ are given by (1.2). Furthermore, the well-known matrix elements of the operators $T(g)$ in the $\left\{f_{m}^{(\ell)}\right\}$ basis can be used to expand the function $\boldsymbol{T}(g) \Psi_{M}^{(L)}$ in terms of the spherical wave basis, [14]. It is easy to show that the recurrence relations (3.12), (3.17) are also satisfied by the non-Hilbert space solutions

$$
\Psi_{m}^{\prime(\ell)}(\rho, \theta, \varphi)=\left(\frac{8 \pi^{3}}{\omega \rho}\right)^{1 / 2} i^{\ell} J_{-\ell-1 / 2}(\omega \rho) Y_{\ell}^{m}(\theta, \varphi)
$$

so the same matrix elements can be used to expand $T(g) \Psi_{M}^{\prime(L)}$ in terms of the functions $\Psi_{m}^{\prime(\ell)}$.

Next we compute the spectral resolutions of the operators corresponding to systems 1]-4] on Table 1 , via our $L_{2}\left(S_{2}\right)$ model. These systems are characterized by the fact that $P_{3}$ is diagonal. From (3.6) it follows that the self-adjoint operator $i P_{3}=-\omega \cos \theta$ has continuous spectrum covering the interval $[-\omega, \omega]$ with multiplicity one. The complete results are:

1] Cartesian system.

The eigenvalue equations

$$
i P_{3} f_{\alpha, r}^{(1)}=-\omega \cos \gamma f_{\alpha, r}^{(1)}, \quad i P_{2} f_{\alpha, r}^{(1)}=-\omega \sin \gamma \sin \alpha f_{\alpha, r}^{(1)}
$$

lead to eigenfunctions

$$
\begin{aligned}
f_{\alpha, \gamma}^{(1)}(\theta, \varphi)= & \frac{\delta(\varphi-\alpha) \delta(\theta-\gamma)}{\sqrt{\sin \gamma}}, \quad-\pi \leq \alpha<\pi, \quad 0 \leq \gamma \leq \pi \\
& \left\langle f_{\alpha, r}^{(1)}, f_{\alpha^{\prime}, \gamma^{\prime}}^{(1)}\right\rangle=\delta\left(\alpha-\alpha^{\prime}\right) \delta\left(\gamma-\gamma^{\prime}\right) .
\end{aligned}
$$

The corresponding solutions of the Helmholtz equation are the plane waves

$$
\begin{aligned}
\Psi_{\alpha \gamma}^{(1)}(\boldsymbol{x}) & =I\left(f_{\alpha \gamma}^{(1)}\right) \\
& =\sqrt{\sin \gamma} \exp \left[i \omega\left(x_{1} \sin \gamma \cos \alpha+x_{2} \sin \gamma \sin \alpha+x_{3} \cos \gamma\right)\right]
\end{aligned}
$$

2] Cylindrical system.

The eigenvalue equations are 


$$
i P_{3} f_{n, r}^{(2)}=-\omega \cos \gamma f_{n, r}^{(2)}, \quad i J_{3} f_{n, r}^{(2)}=n f_{n, r}^{(2)}
$$

and the basis of eigenfunctions is

$$
\begin{gathered}
f_{n, r}^{(2)}(\theta, \varphi)=\frac{e^{i n \varphi} \delta(\gamma-\theta)}{\sqrt{2 \pi \sin \gamma}}, \quad n=0, \pm 1, \cdots, 0 \leq \gamma \leq \pi, \\
\left\langle f_{n, \gamma}^{(2)}, f_{n^{\prime} \gamma^{\prime}}^{(2)}\right\rangle=\delta_{n n^{\prime} \delta}\left(\gamma-\gamma^{\prime}\right) .
\end{gathered}
$$

Furthermore,

$$
\Psi_{n, r}^{(2)}(\boldsymbol{x})=i^{n} \sqrt{2 \pi \sin \gamma} J_{n}(\omega \sin \gamma r) \exp [i(n \varphi+\omega z \cos \gamma)] .
$$

These are cylindrical wave solutions of the Helmholtz equation.

3] Parabolic cylindrical system.

The eigenvalue equations are

$$
i P_{3} f_{\mu \pm, r}^{(3)}=-\omega \cos \gamma f_{\mu \pm, r}^{(3)}, \quad\left\{J_{3}, P_{2}\right\} f_{\mu \pm, r}^{(3)}=2 \mu \omega \sin \gamma f_{\mu \pm, r}^{(3)}
$$

and the basis of eigenfunctions is

$$
\left.\begin{array}{rl}
f_{\mu+, \gamma}^{(3)}(\theta, \varphi) & = \begin{cases}\frac{1}{\sqrt{2 \pi \sin \gamma}}(1+\cos \varphi)^{-i \mu / 2-1 / 4}(1-\cos \varphi)^{i \mu / 2-1 / 4} \mathscr{S}(\theta-\gamma), \\
0, \quad-\pi<\varphi<0\end{cases} \\
f_{\mu-, \gamma}^{(3)}(\theta, \varphi) & =f_{\mu+, \gamma}^{(3)}(\theta,-\varphi), \quad-\infty<\mu<\infty, \quad 0 \leq \gamma \leq \pi,
\end{array}\right\}
$$

The corresponding solutions of (0.1) are

$$
\begin{aligned}
\Psi_{\mu, \gamma}^{(3)}(x)= & \sqrt{\frac{\sin \gamma}{2}} \sec (i \mu \pi) \exp (i \omega z \cos \gamma) \\
& \times\left[D_{i \mu-1 / 2}(\sigma \xi) D_{-i \mu-1 / 2}(\sigma \eta)+D_{i \mu-1 / 2}(-\sigma \xi) D_{-i \mu-1 / 2}(-\sigma \eta)\right] \\
\Psi_{\mu-, \gamma}^{(3)}(\xi, \eta, z)= & \Psi_{\mu+, \gamma}^{(3)}(\xi,-\eta, z) \\
\sigma= & e^{i \pi / 4} \sqrt{2 \omega \sin \gamma} .
\end{aligned}
$$

4] Elliptic cylindrical system.

The eigenvalue equations are

$$
i P_{3} f_{n t, r}^{(4)}=-\omega \cos \gamma f_{n t, r}^{(4)}, \quad\left(J_{3}^{2}+d^{2} P_{1}^{2}\right) f_{n t, r}^{(4)}=\lambda_{n t} f_{n t, r}^{(4)}, \quad t=s, c,
$$

and the basis of eigenfunctions is

$$
f_{n c, \gamma}^{(4)}(\theta, \varphi)=\frac{1}{\sqrt{\pi \sin \varphi}} c e_{n}(\varphi, q) \delta(\theta-\gamma), \quad n=0,1,2, \cdots
$$




$$
\begin{aligned}
f_{n s, \gamma}^{(4)}(\theta, \varphi) & =\frac{1}{\sqrt{\pi \sin \varphi}} \operatorname{se}_{n}(\varphi, q) \delta(\theta-\gamma), \quad n=1,2, \ldots \\
q & =d^{2} \omega^{2} \sin ^{2} \gamma / 4, \quad 0 \leq \gamma \leq \pi .
\end{aligned}
$$

The eigenvalues $\lambda_{n t}$ are discrete, of multiplicity one, and related to the eigenvalues $a$ of Mathieu's equations, [10], by $a=-\lambda-\frac{1}{2} d^{2} \omega^{2} \sin ^{2} \gamma$. The eigenvectors form a basis for $L_{2}\left(S_{2}\right)$ satisfying

$$
\left\langle f_{n t, r}^{(4)}, f_{n^{\prime} t^{\prime}, r^{\prime}}^{(4)}\right\rangle=\delta_{n n^{\prime}} \delta_{t t^{\prime}} \delta\left(\gamma-\gamma^{\prime}\right), \quad t, t^{\prime}=s, c,
$$

and the corresponding solutions of (0.1) are

$$
\begin{aligned}
& \Psi_{n c, \gamma}^{(4)}(\boldsymbol{x})=C_{n} \sqrt{\sin \gamma} C e_{n}(\alpha, q) c e_{n}(\beta, q) \exp (i w z \cos \gamma) \\
& n=0,1,2, \cdots, \\
& \Psi_{n s, r}^{(4)}(\boldsymbol{x})=S_{n} \sqrt{\sin \gamma} S_{n}(\alpha, q) e_{n}(\beta, q) \exp (i \omega z \cos \gamma) \\
& n=1,2, \cdots,
\end{aligned}
$$

where $S \mathrm{e}_{n}, C e_{n}$ are associated Mathieu functions and $S_{n}, C_{n}$ are constants to be determined from the integral equation $\Psi_{n t, r}^{(4)}=I\left(f_{n t, r}^{(4)}\right)$.

6] Prolate spheroidal system.

The eigenvalue equations are

$$
\left(\boldsymbol{J} \cdot \boldsymbol{J}-a^{2} P_{1}^{2}-a^{2} P_{2}^{2}\right) f_{n, m}^{(6)}=-\lambda_{n}^{m} f_{n, m}^{(6)}, \quad i J_{3} f_{n, m}^{(6)}=m f_{n, m}^{(6)}
$$

and the basis of eigenfunctions is

$$
f_{n, m}^{(6)}(\theta, \varphi)=\left[\frac{(2 n+1)(n-|m|) !}{4 \pi(n+|m|) !}\right]^{1 / 2} P s_{n}^{|m|}\left(\cos \theta, a^{2} \omega^{2}\right) e^{i m \varphi}
$$

(The first eigenvalue equation (3.33) takes the place of the second equation (2.13).) Here $n=0,1,2, \cdots, m=n, n-1, \cdots,-n$ and the discrete eigenvalues are denoted $\lambda_{n}^{m}\left(\alpha^{2} \omega^{2}\right)$. We have $\left\langle f_{m, n}^{(6)}, f_{n^{\prime}, m^{\prime}}^{(6)}\right\rangle=\delta_{n n^{\prime}} \delta_{m m}$. The spheroidal wave functions are frequently defined by their expansions in terms of associated Legendre functions:

$$
P S_{n}^{|m|}\left(x, a^{2} \omega^{2}\right)=\sum_{2 k \geq|m|-n}(-1)^{\ell} a_{n, 2 k}^{|m|}\left(a^{2} \omega^{2}\right) P_{n+2 k}^{|m|}(x),
$$

see [10], page 169. Indeed, substituting (3.25) into the spheroidal wave equation one can derive a recurrence formula for the coefficients $a_{n, 2 k}^{|m|}$.

The corresponding basis of solutions for (0.1) is

$$
\Psi_{n, m}^{(6)}(\boldsymbol{x})=c_{n}^{m}\left(a^{2} \omega^{2}\right) S_{n}^{m(1)}(\cosh \eta, a \omega) P s_{n}^{|m|}\left(\cos \alpha, a^{2} \omega^{2}\right) e^{i m \varphi}
$$


where $c_{n}^{m}\left(a^{2} \omega^{2}\right)$ is a constant to be determined from the integral equation. This result is easily obtained from the fact that $\Psi_{n, m}^{(6)}$ must be separable in prolate spheroidal coordinates.

7] Oblate spheroidal system.

The eigenvalue equations are

$$
\left(\boldsymbol{J} \cdot \boldsymbol{J}+a^{2} P_{1}^{2}+a^{2} P_{2}^{2}\right) f_{n, m}^{(7)}=-\lambda_{n}^{m} f_{n, m}^{(7)}, \quad i J_{3} f_{n, m}^{(7)}=m f_{n, m}^{(7)}
$$

and the orthonormal basis of eigenfunctions is

$$
\begin{aligned}
f_{n, m}^{(7)}(\theta, \varphi)=\left[\frac{(2 n+1)(n-|m|) !}{4 \pi(n+|m|) !}\right]^{1 / 2} P s_{n}^{|m|}\left(\cos \theta,-\alpha^{2} \omega^{2}\right) e^{i m \varphi} \\
n=0,1,2, \cdots, m=n, n-1, \cdots,-n .
\end{aligned}
$$

The discrete eigenvalues are $\lambda_{n}^{|m|}\left(-a^{2} \omega^{2}\right)$. The corresponding solutions of the Helmholtz equation are

$$
\Psi_{n, m}^{(7)}(\boldsymbol{x})=c_{n}^{m}\left(a^{2} \omega^{2}\right) S_{n}^{m(1)}(-i \sinh \eta, i a \omega) P s_{n}^{|m|}\left(\cos \alpha,-a^{2} \omega^{2}\right) e^{i m \varphi}
$$

where $c_{n}^{m}\left(a^{2} \omega^{2}\right)$ is a constant to be determined from the integral equation.

8] Parabolic system.

The eigenvalue equations are

$$
\left(\left\{J_{1}, P_{2}\right\}-\left\{J_{2}, P_{1}\right\}\right) f_{\lambda, m}^{(8)}=2 \lambda \omega f_{\lambda, m}^{(8)}, \quad i J_{3} f_{\lambda, m}^{(8)}=m f_{\lambda, m}^{(8)},
$$

see [13]. Here $\left\{J_{1}, P_{2}\right\}-\left\{J_{2}, P_{1}\right\}=2 i \omega\left(\cos \theta+\sin \theta \partial_{\theta}\right)$ is first-order and has a unique self-adjoint extension. The eigenfunctions are

$$
\begin{aligned}
f_{\lambda, m}^{(8)}(\theta, \varphi) & =\frac{[\tan (\theta / 2)]^{-i \lambda}}{\sin \theta} \frac{e^{i m \varphi}}{2 \pi}, \quad m=0, \pm 1, \cdots,-\infty<\lambda<\infty, \\
\left\langle f_{\lambda, m}^{(8)}, f_{\lambda^{\prime}, m^{\prime}}^{(8)}\right\rangle & =\delta\left(\lambda-\lambda^{\prime}\right) \delta_{m m^{\prime}}
\end{aligned}
$$

The corresponding solutions of (0.1) are

$$
\begin{gathered}
\Psi_{\lambda, m}^{(8)}(\boldsymbol{x})=\frac{i^{m} \sqrt{\mathbf{2}}}{\xi \eta \omega} \Gamma\left(\frac{1-m}{2}+i \lambda\right) \Gamma\left(\frac{1-m}{2}-\frac{i \lambda}{2}\right) \\
\mathscr{M}_{i \lambda / 2,-m / 2}\left(e^{-i \pi / 2} \omega \xi^{2} / \sqrt{\mathbf{2}}\right) \mathscr{M}_{i \lambda / 2,-m / 2}\left(e^{i \pi / 2} \omega \eta^{2} / \sqrt{\mathbf{2}}\right) e^{i m \varphi}
\end{gathered}
$$

where

$$
\mathscr{M}_{\alpha, \mu / 2}(z)=\frac{z^{(1+\mu) / 2} e^{-z / 2}}{\Gamma(1+\mu)}{ }_{1} F_{1}\left(\begin{array}{c}
(1+\mu) / 2-\alpha \\
1+\mu
\end{array} \mid-z\right) .
$$


9] Paraboloidal system.

The eigenvalue equations are

$$
\begin{aligned}
& \left(J_{3}^{2}-c^{2} P_{3}^{2}+c\left\{J_{2}, P_{1}\right\}+c\left\{J_{1}, P_{2}\right\}\right) f_{n t, \lambda}^{(9)}=-\mu_{n t} f_{n t, \lambda}^{(9)} \\
& \left(c P_{2}^{2}-c P_{1}^{2}+\left\{J_{2}, P_{1}\right\}-\left\{J_{1}, P_{2}\right\}\right) f_{n t, \lambda}^{(9)}=2 \omega \lambda f_{n t, \lambda}^{(9)}
\end{aligned}
$$

and the basis of eigenfunctions is

$$
\begin{aligned}
f_{n t, \lambda}^{(9)}(\theta, \varphi) & =\frac{(\tan \theta / 2)^{i \lambda}}{\sqrt{2 \pi} \sin \theta} \exp \left(\frac{i c \omega}{2} \cos \theta \cos 2 \varphi\right) \times\left\{\begin{array}{l}
g c_{n}(\varphi, 2 c \omega, \lambda) \\
g s_{n}(\varphi, 2 c \omega, \lambda)
\end{array}\right. \\
t & =c, s, \quad n=0,1,2, \cdots,-\infty<\lambda<\infty,
\end{aligned}
$$

where $g c_{n}$ and $g s_{n}$ are the even and odd nonpolynomial solutions of the Whittaker-Hill equation, [12]. We have

$$
\left\langle f_{n t, \lambda}^{(9)}, f_{n^{\prime} t^{\prime}, \lambda^{\prime}}^{(9)}\right\rangle=\delta_{n n^{\prime}}, \delta_{t t^{\prime}} \delta\left(\lambda-\lambda^{\prime}\right) \text {. }
$$

The corresponding solutions of (0.1) are

$$
\begin{array}{r}
\Psi_{n t, \lambda}^{(9)}(\boldsymbol{x})=K_{n}^{t}(\omega c, \lambda) g t_{n}(\beta, 2 c \omega, \lambda) g t_{n}(i \alpha, 2 c \omega, \lambda) g t_{n}(i \gamma+\pi / 2,2 c \omega, \lambda), \\
t=s, c,
\end{array}
$$

where the constants $K_{n}^{t}$ are to be determined from the integral equation $\Psi_{n t, \lambda}^{(9)}=I\left(f_{n t, \lambda}^{(9)}\right)$. This equation appears to be a new result.

10] Ellipsoidal system.

Here we adopt elliptic coordinates $(s, t)$ on $S_{2}$ :

$$
\begin{gathered}
k_{1}=\left[\frac{(s-a)(t-a)}{a(a-1)}\right]^{1 / 2}, \quad k_{2}=\left[\frac{(s-1)(t-1)}{1-a}\right]^{1 / 2}, k_{3}=\left[\frac{s t}{a}\right]^{1 / 2} \\
0<t<1<s<a .
\end{gathered}
$$

Then the eigenvalue equations

$$
\begin{gathered}
S f=\lambda f, \quad S^{\prime} f=\mu f \\
S=P_{1}^{2}+a P_{2}^{2}+(a+1) P_{3}^{2}+J \cdot J, \quad S^{\prime}=J_{2}^{2}+a J_{1}^{2}+a P_{3}^{2}
\end{gathered}
$$

become

$$
\left[\frac{4}{s-t}\left(\partial_{\alpha \alpha}+\partial_{\beta \beta}\right)-\omega^{2}(s+t)-\omega^{2}(1+a)\right] f=\lambda f
$$

$$
\begin{aligned}
& {\left[\frac{4}{s-t}\left(t \partial_{\alpha \alpha}+s \partial_{\beta \beta}\right)-\omega^{2} s t\right] f=\mu f} \\
& \partial_{\alpha}=[(a-s)(s-1) s]^{1 / 2} \partial_{s}, \quad \partial_{\beta}=[(t-a)(t-1) t]^{1 / 2} \partial_{t} .
\end{aligned}
$$

We can find solutions of these equations in the form $f(x, t)=E_{1}(s) E_{2}(t)$ where 


$$
\begin{aligned}
& \left(4 \partial_{\alpha \alpha}-\omega^{2} s^{2}+\lambda^{\prime} s+\mu\right) E_{1}(s)=0, \quad \lambda^{\prime}=-\omega^{2}(1+a)-\lambda, \\
& \left(4 \partial_{\beta \beta}+\omega^{2} t^{2}-\lambda^{\prime} t-\mu\right) E_{2}(t)=0 .
\end{aligned}
$$

Expressions (3.49) are algebraic forms of the ellipsoidal wave equation so the $E_{j}$ are ellipsoidal functions. Furthermore, if we set $s=s^{2}(\eta, k)$, $t=\operatorname{sn}^{2}(\psi, k)$ where $k=a^{-1 / 2}$ then the separated equations take the Jacobian form

$$
\left(\partial_{\xi \xi}-k^{2} \mu-k^{2} \lambda^{\prime} s n^{2} \xi+k^{2} \omega^{2} s n^{4} \xi\right) E_{j}(\xi)=0, \quad \xi=\eta, \psi, \quad j=1,2
$$

of the ellipsoidal wave equation (2.29). The new coordinates $\eta, \psi$ also have the property that they allow us to parametrize the entire sphere $S_{2}$ rather than just the first octant. Indeed,

$$
k_{1}=k^{\prime-1} d n \eta d n \psi, \quad k_{2}=i k k^{\prime-1} c n \eta c n \psi, \quad k_{3}=k s n \eta s n \psi
$$

and these coordinates cover $S_{2}$ exactly once if $\eta \in[-2 K, 2 K], \psi \in[K, K+$ $\left.2 i K^{\prime}\right]$.

Since $k_{1}, k_{2}, k_{3}$ remain unchanged when integral multiples of $4 K$ and $4 i K^{\prime}$ are added to $\eta$ or $\psi$, we are interested only in those single-valued solutions $E_{j}$ of (3.50) which are also fixed under these substitutions: $E_{\jmath}\left(\xi+4 K_{n}+4 i K^{\prime} m\right)=E_{\jmath}(\xi), n, m$ integers. As we noted in Section 2 , these doubly-periodic functions are the ellipsoidal wave functions. They have been studied in detail by Arscott, [10]. The spectrum of $S$ and $S^{\prime}$ is discrete, each pair of eigenvalues denoted $\lambda_{n m}, \mu_{n m}$. The corresponding ellipsoidal wave functions are $e \ell_{n}^{m}(\xi), \xi=\eta, \psi$ and the eigenfunctions of $S$ and $S^{\prime}$ are denoted

$$
f_{n, m}^{(10)}(\eta, \psi)=e \ell p_{n}^{m}(\eta, \psi)=e \ell_{n}^{m}(\eta) e \ell_{n}^{m}(\psi)
$$

where $n=0,1,2, \cdots$ and the integer $m$ runs over $2 n+1$ values. We assume that the basis $\left\{e \ell p_{n}^{m}\right\}$ is normalized so that

$$
\left\langle e \ell p_{n}^{m}, e \ell p_{n^{\prime}}^{m^{\prime}}\right\rangle=\delta_{n n^{\prime}} \delta_{m m^{\prime}} \text {. }
$$

Note that

$$
d \Omega(\boldsymbol{k})=i k^{2}\left(s n^{2} \eta-s n^{2} \psi\right) d \eta d \psi
$$

In general these functions are rather intractible and very little is known about their explicit construction.

The corresponding solutions of the Helmholtz equation $\Psi_{n m}^{(10)}(\boldsymbol{x})=$ $I\left(f_{n m}^{(10)}\right)$ are 


$$
\Psi_{n m}^{(10)}(\boldsymbol{x})=E \ell_{n}^{m}(\alpha, \beta, \gamma)=K_{n}^{m}(\omega, k) e \ell_{n}^{m}(\alpha) e \ell_{n}^{m}(\beta) e \ell_{n}^{m}(\gamma)
$$

where the constant $K_{n}^{m}(\omega, k)$ is to be evaluated from the integral equation. Moreover, the equation reads

$$
\begin{aligned}
E \ell_{n}^{m}(\alpha, \beta, \gamma)= & i k^{2} \int_{S_{2}} \exp \left[\omega \left(-\frac{1}{k k^{\prime 2}} d n \alpha d n \beta d n \gamma d n \eta d n \psi\right.\right. \\
& \left.\left.+\frac{k^{2}}{k^{\prime 2}} c n \alpha c n \beta c n \gamma c n \eta c n \psi+i k^{2} \operatorname{sn} \alpha \operatorname{sn} \beta \operatorname{sn} n s n \psi\right)\right] \\
& \times \operatorname{elp}(\eta, \psi)\left(s n^{2} \eta-s n^{2} \psi\right) d \eta d \psi,
\end{aligned}
$$

which appears to be a new result. We were able to evaluate the integral (3.54) to within a constant multiple because we knew in advance that it was separable in $\alpha, \beta, \gamma$.

11] Conical system.

Only for the conical and spherical systems does the eigenvalue problem become finite-dimensional. For $f \in L_{2}\left(S_{2}\right)$ the eigenvalue problem associated with system 11] is

$$
\boldsymbol{J} \cdot \boldsymbol{J} \boldsymbol{f}=-\ell(\ell+1) f, \quad\left(J_{1}^{2}+b J_{2}^{2}\right) f=\lambda f, \quad 1>b>0 .
$$

From (3.13) the eigenvalue problem reduces to computing the eigenvalues of $J_{1}^{2}+b J_{2}^{2}$ on the $(2 \ell+1)$-dimensional space $V_{\ell},(3.12)$. This problem is solved in [15] and [16] where the relationship between the spherical and conical bases is derived. However, equation (5.16) in [16] is in error and will be corrected in [17].

\section{Section 4. Expansion formulas for solutions of the Helmholtz equation.}

From (3.11) it is evident that if we wish to expand a solution $\boldsymbol{T}(g) \Psi_{\lambda}^{(j)}$ of (0.1) in terms of eigenfunctions $\left\{\Psi_{\mu}^{(i)}\right\}$ we can do so by computing the expansion coefficients $\left\langle\boldsymbol{T}(g) f_{\lambda}^{(j)}, f_{\mu}^{(i)}\right\rangle$ in the $L_{2}\left(S_{2}\right)$ model:

$$
\boldsymbol{T}(g) \Psi_{\lambda}^{(j)}(\boldsymbol{x})=\sum_{\mu}\left\langle\boldsymbol{T}(g) f_{\lambda}^{(j)}, f_{\mu}^{(i)}\right\rangle \Psi_{\mu}^{(i)}(\boldsymbol{x})
$$

Here we list some of the more tractible expansion coefficients in the case where $\boldsymbol{T}(g)$ is the identity operator.

The overlap functions $\left\langle f_{\lambda}^{(j)}, f_{\alpha, \gamma}^{(1)}\right\rangle$ relating any system $\left\{f_{\lambda}^{(j)}(\boldsymbol{k})\right\}$ with the Cartesian system (3.21) are trivial:

$$
\left\langle f_{\lambda}^{(j)}, f_{\alpha, \gamma}^{(1)}\right\rangle=\sqrt{\sin \gamma} f_{\lambda}^{(j)}(\sin \gamma \cos \alpha, \sin \gamma \sin \alpha, \cos \gamma) \text {. }
$$


Moreover, the overlaps relating the eigenfunctions for systems 1]-4] on Table 1 can easily be obtained from the corresponding overlaps for solutions of the Helmholtz equation $\left(\Delta_{2}+\omega^{2}\right) \psi=0$ listed in [2]. Indeed the results take the form

$$
\left\langle f_{\lambda, r}^{(j)}, f_{\mu, r^{\prime}}^{(i)}\right\rangle=\delta\left(\gamma-\gamma^{\prime}\right)\left\langle f_{\lambda}^{(j)^{\prime}}, f_{\mu}^{(i)^{\prime}}\right\rangle
$$

where $\left\langle f_{\lambda}^{(j)^{\prime}}, f_{\mu}^{(i)^{\prime}}\right\rangle$ is the corresponding overlap in [2] with the $L_{2}[-\pi, \pi]$ model.

The overlaps between the spherical and parabolic bases were computed in [13]:

$$
\begin{aligned}
&\left\langle f_{m}^{(\ell)}, f_{\lambda, m^{\prime}}^{(8)}\right\rangle= \delta_{m m^{\prime}} \frac{(-1)^{(m+|m|) / 2}}{(|m| !)^{2}}\left[\frac{(2 \ell+1)(\ell+|m|) !}{4 \pi(\ell-|m|) !}\right]^{1 / 2} \\
& \times \Gamma\left(\frac{i \lambda+|m|+1}{2}\right) \Gamma\left(\frac{-i \lambda+|m|+1}{2}\right) \\
&{ }_{3} F_{2}(|m|-\ell,|m|+\ell+1,(i \lambda+|m|+1) / 2 \mid 1), \\
&|m|+1,|m|+1 \quad m=0, \pm 1, \cdots, \pm \ell .
\end{aligned}
$$

The overlaps between the spherical and prolate spheroidal bases are

$$
\left\langle f_{m}^{(\ell)}, f_{n, m^{\prime}}^{(6)}\right\rangle=\left\{\begin{array}{rr}
\delta_{m m^{\prime}}(-1)^{(2 m+\ell-n) / 2}\left[\frac{(n-m) !(\ell+m) !}{(n+m) !(\ell-m) !} \frac{2 n+1}{2 \ell+1}\right]^{1 / 2} \\
\times a_{n, \ell-n}^{m}\left(a^{2} \omega^{2}\right), \quad m^{\prime} \geq 0 \\
\delta_{m m^{\prime}}(-1)^{(\ell-2) / 2}\left[\frac{(n+m) !}{(n-m) !} \frac{2 n+1}{2 \ell+1}\right]^{1 / 2} a_{n, \ell-n}^{|m|}\left(a^{2} \omega^{2}\right), \\
m^{\prime}<0
\end{array}\right.
$$

where the coefficients $a_{n, 2 k}^{|m|}$ are defined by (3.35).

The overlaps between the cylindrical and prolate spheroidal bases are

$$
\left\langle f_{n, m}^{(6)}, f_{m^{\prime}, r}^{(2)}\right\rangle=\left[\frac{(n-|m|) !}{(n+|m|) !} \frac{2 n+1}{2} \sin \gamma\right]^{1 / 2} P s_{n}^{|m|}\left(\cos \gamma, a \omega^{2}\right) \delta_{m m^{\prime}}
$$

and the overlaps between the parabolic cylindrical and prolate spheroidal bases are

$$
\begin{aligned}
\left\langle f_{n, m}^{(6)}, f_{\mu \pm, r}^{(3)}\right\rangle= & {\left[\frac{(n-|m|) !}{(n+|m|) !} \frac{(2 n+1)}{(2)} \sin \gamma\right]^{1 / 2} } \\
& \times P s_{n}^{|m|}\left(\cos \gamma, a^{2} \omega^{2}\right)\left\langle f_{m}^{(2)}, f_{\mu \pm}^{(3)}\right\rangle
\end{aligned}
$$

where the overlap $\left\langle f_{m}^{(2)}, f_{ \pm \mu}^{(3)}\right\rangle$ in $L_{2}[-\pi, \pi]$ is computed in [2] and [18]. 
The overlaps between the elliptic cylindrical and prolate spheroidal bases are

$$
\left\langle f_{n, m}^{(6)}, f_{n^{\prime} \rho, \gamma}^{(4)}\right\rangle=\left[\frac{(n-|m|) !}{(n+|m|) !}(2 m+1) \sin \gamma\right]^{1 / 2} P s_{n}^{|m|}\left(\cos \gamma, a^{2} \omega^{2}\right) A_{n^{\prime}}^{m}
$$

where the Fourier coefficient $A_{n}^{m}$ is defined in terms of the Mathieu functions $p e_{n}(\varphi, q), p=s, c$, by

$$
p e_{n}(\varphi, q)=\sum_{m=-\infty}^{\infty} A_{n}^{m} e^{i m \varphi} \text {. }
$$

The corresponding overlaps for oblate spheroidal coordinates can be obtained from the prolate overlaps (4.5)-(4.8) by making the replacement $a^{2} \omega^{2} \rightarrow-a^{2} \omega^{2}$ in the speroidal wave function.

The overlaps between the spherical and conical bases are computed in [15], [16] and those between the ellipsoidal and conical bases are discussed in [10], page 247.

The remaining overlaps are more complicated than those we have listed.

It is easy to construct a bilinear generating function for all basis sets of solutions of (0.1) listed here. Let $\left\{f_{\lambda \mu}(\boldsymbol{k})\right\}$ be one of the eleven bases for $L_{2}\left(S_{2}\right)$ constructed above and let $\left\{\Psi_{\lambda \mu}(x)\right\}$ be the corresponding basis for the solution space of (0.1). Then

$$
\Psi_{\lambda \mu}(\boldsymbol{x})=I\left(f_{\lambda \mu}\right)=\left\langle f_{\lambda \mu}, H(\boldsymbol{x}, \cdot)\right\rangle
$$

where $H(\boldsymbol{x}, \cdot) \in L_{2}\left(S_{2}\right)$, (3.8), for each $\boldsymbol{x} \in \boldsymbol{R}^{3}$. An explicit computation yields

$$
\left\langle H(x, \cdot), H\left(x^{\prime}, \cdot\right)\right\rangle=4 \pi \frac{\sin (\omega R)}{\omega R}, \quad R^{2}=\left(x-x^{\prime}\right) \cdot\left(x-x^{\prime}\right) .
$$

On the other hand

$$
\begin{aligned}
\left\langle H(\boldsymbol{x}, \cdot), H\left(\boldsymbol{x}^{\prime}, \cdot\right)\right\rangle & =\sum_{\lambda, \mu}\left\langle H(\boldsymbol{x}, \cdot), f_{\lambda \mu}\right\rangle\left\langle f_{\lambda \mu}, H\left(\boldsymbol{x}^{\prime}, \cdot\right)\right\rangle \\
& =\sum_{\lambda, \mu} \bar{\Psi}_{\lambda \mu}(\boldsymbol{x}) \Psi_{\lambda \mu}\left(\boldsymbol{x}^{\prime}\right)
\end{aligned}
$$

and comparison of $(4.10),(4.11)$ shows that $4 \pi \sin (\omega R) / \omega R$ is a bilinear generating function for each of our bases.

Finally, as shown in [8] and [13], each of our eleven bases $\left\{\Psi_{\lambda \mu}\right\}$ considered as functions of $\omega, 0<\omega<\infty$, can be used to expand arbitrary functions $f \in L_{2}\left(R^{3}\right)$. 


\section{Section 5. Non-Hilbert space models.}

Obviously, many separable solutions of (0.1) are not representable in the form $I(h)$, (2.1), for $h \in L_{2}\left(S_{2}\right)$. We shall investigate a few grouptheoretic methods for obtaining such solutions and relating different types of separable non-Hilbert space solutions. These methods are less elegant but more flexible than the techniques discussed earlier.

We begin by considering transforms (2.1) where the domain of integration is a complex two-dimensional Riemann surface rather than the real sphere $S_{2}$. In particular we set

$$
\boldsymbol{k}=\left(k_{1}, k_{2}, k_{3}\right)=\left(-\frac{1}{2}\left(t-t^{-1}\right) \sqrt{1+\beta^{2}}, \frac{i}{2}\left(t-t^{-1}\right) \sqrt{1+\beta^{2}}, i \beta\right)
$$

where $t$ and $\beta$ range over complex values, and write

$$
\begin{aligned}
\Psi(x)= & \int_{S} d \beta \frac{d t}{t} h(\beta, t) \exp \left[-\frac{i \omega}{2} x\left(t+t^{-1}\right) \sqrt{1+\beta^{2}}\right. \\
& \left.+\frac{\omega y}{2}\left(t^{-1}-t\right) \sqrt{1+\beta^{2}}-\omega \beta z\right]=I(h) .
\end{aligned}
$$

We assume that the integration surface $S$ and the analytic function $h$ are such that $I(h)$ converges absolutely and arbitrary differentiation with respect to $x, y, z$ is permitted under the integral sign. Since $\boldsymbol{k} \cdot \boldsymbol{k}$ $=1$ even for arbitrary complex $\beta, t(t \neq 0)$, it follows that $\Psi(x)$ is a solution of $(0.1)$.

Integrating by parts, we find that the operators $P_{j}, J_{j}$ (1.2), acting on the solution space of $(0.1)$ correspond to the operators

$$
\begin{gathered}
J^{ \pm}=i t^{ \pm 1}\left(\mp \sqrt{1+\beta^{2}} \partial_{\beta}+\frac{\beta t}{\sqrt{1+\beta^{2}}} \partial_{t}\right) \\
J^{0}=t \partial_{t}, \quad P^{ \pm}=\omega \sqrt{1+\beta^{2}} t^{ \pm 1}, \quad P^{0}=-i \omega \beta
\end{gathered}
$$

acting on the analytic functions $h(\beta, t)$, provided $S$ and $h$ are chosen such that the boundary terms vanish:

$$
J^{ \pm} \Psi=I\left(J^{ \pm} h\right), \quad P^{ \pm} \Psi=I\left(P^{ \pm} h\right),
$$

etc. Here $J^{ \pm}=\mp J_{2}+i J_{1}, J^{0}=i J_{3}, P^{ \pm}=\mp P_{2}+i P_{1}, P^{0}=i P_{3}$.

For our first example we set $h=\left(2 \pi^{3}\right)^{-1 / 2}$ and integrate over the contours $C_{1}$ and $C_{2}$ in the $\beta$ and $t$ planes, respectively. 


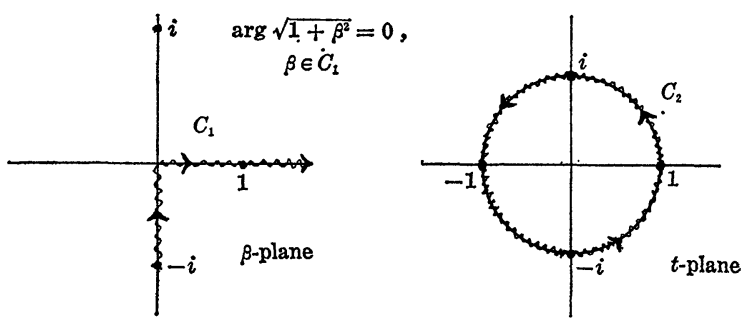

Figure 1

In this case $h$ satisfies the equations $\boldsymbol{J} \cdot \boldsymbol{J} h=0, J^{0} h=0$ and it is straightforward to verify that $\Psi(x)=I(h)$ satisfies the same equations for $z>0$. Thus, $\Psi$ is independent of the spherical coordinates $\theta, \varphi$ and is a linear combination of the Bessel functions (2.11) for $l=0$. To determine the correct linear combination we evaluate (5.2) in the special case $x=y=$ 0 . The integral is elementary and we find

$$
\Psi(0,0, z)=i \sqrt{\frac{2}{\pi}} \frac{e^{i \omega z}}{\omega z}, \quad z>0
$$

so

$$
\Psi(x)=-(\omega \rho)^{-1 / 2} H_{1 / 2}^{(1)}(\omega \rho)
$$

whose $H_{\nu}^{(1)}(z)$ is a Hankel function of the first kind, [11]. Solution (5.4) is a (traveling) spherical wave.

More generally we set

$$
\begin{aligned}
h=f_{m}^{(\ell)}(\beta, t)=\left[\frac{(2 \ell+1)(\ell-m) !}{4 \pi(\ell+m) !}\right]^{1 / 2} P_{\ell}^{m}(-\beta)(-t)^{m} \\
\quad \ell=0,1, \cdots, m=\ell, \ell-1, \cdots,-\ell .
\end{aligned}
$$

It is straightforward to verify that these functions and the operators (5.3) satisfy the recurrence formulas (3.12), (3.17). Thus, the solutions $\Psi_{m}^{(\ell)}(\boldsymbol{x})=I\left(f_{m}^{(\ell)}\right)$ also satisfy these relations. We have already computed $\Psi_{0}^{(0)}(\boldsymbol{x})$, and using these recurrence relation, we can obtain

$$
\Psi_{m}^{(\ell)}(\rho, \theta, \varphi)=-(i)^{\ell}(\omega \rho)^{-1 / 2} H_{\ell+1 / 2}^{(1)}(\omega \rho) Y_{\ell}^{m}(\theta, \varphi)
$$

Next we consider the cylindrical system corresponding to the operators (5.3):

(5.7) $\quad P^{0} f_{m, r}^{(2)}=i \omega \gamma f_{m, r}^{(2)}, \quad J^{0} f_{m, r}^{(2)}=m f_{m, r}^{(2)}, \quad f_{m, r}^{(2)}(\beta, t)=\delta(\beta-\gamma) t^{m}$.

Using the contours $C_{1}, C_{2}$ we find

$$
\Psi_{m . r}^{(2)}(r, \theta, z)=i^{m+1}(-1)^{m}(2 \pi) J_{m}\left(\omega r \sqrt{\left.1+\gamma^{2}\right)} e^{i m \theta-\omega \gamma z}\right.
$$


for $\gamma \in C_{1}$. Here we are using cylindrical coordinates 2]. From (5.5), (5.7) and the corresponding integral representations $\Psi=I(f)$ there follows easily the expansion

$$
\Psi_{m}^{(\ell)}(\boldsymbol{x})=(-1)^{m}\left[\frac{(2 \ell+1)(\ell-m) !}{4 \pi(\ell+m) !}\right]^{1 / 2} \int_{C_{1}} P_{\ell}^{m}(i \beta) \Psi_{m, \beta}^{(2)}(\boldsymbol{x}) d \beta,
$$

More generally, if $\Psi_{m}^{(\ell)}$ is subjected to a translation $\boldsymbol{T}(g)=\exp (\boldsymbol{a} \cdot \boldsymbol{P})$ we obtain

$$
\begin{aligned}
& \boldsymbol{T}(g) \Psi_{m}^{(\ell)}(\boldsymbol{x})=\left[\frac{(2 \ell+1)(\ell-m) !}{4 \pi(\ell+m) !}\right]^{1 / 2} \sum_{n=-\infty}^{\infty} \int_{C_{1}}(-1)^{n+m}\left(i e^{-i \alpha}\right)^{n} \\
& \times P_{\ell}^{m}(i \beta) J_{n}\left(\omega a \sqrt{1+\beta^{2}}\right) e^{-a_{3} \omega \beta} \Psi_{m+n, \beta}^{(2)}(\boldsymbol{x}) d \beta \\
& \\
& z+a_{3}>0, a_{1}+i a_{2}=\alpha e^{i \alpha}, a>0 .
\end{aligned}
$$

Similar techniques can be used to expand traveling spherical waves in other bases. In each case one derives the expansion for the complex sphere model and then attempts to map the results to the solution space of the Helmholtz equation via the transformation (5.2). Some important cases are worked out (by another method) in [19], Section 16.

Other more complicated examples following this approach are worked out in [18] and [13] where bases corresponding to the parabolic cylindrical and the parabolic coordinates are constructed. The basis functions are expressable as products of Hermite polynomials and associated Laguerre polynomials, respectively, and our method allows the simple derivation of addition theorems for these solutions under the action of $E(3)$.

Next we consider identities for solutions of (0.1) which are derivable by Weisner's method, [20], [14]. The natural setting for this method is the complex Helmholtz equation obtained by allowing all variables in (0.1) to assume complex values. To treat this equation systematically we should determine all complex analytic coordinate systems in which variables separate in (0.1). However, here we consider only a few systems of particular importance.

Of greatest practical importance is the spherical system (3.12)'. We now study solutions of the complex equation (0.1) which satisfy (3.12) for general $\ell, m \in C$. In terms of the complex sphere model (5.3) and new complex coordinates $\tau, \rho$

$$
\tau=t \sqrt{1+\beta^{2}}, \quad \rho=-i \beta,
$$

the operators assume the form

$$
\begin{aligned}
& J^{+}=-\tau \partial_{\rho}, J^{-}=\tau^{-1}\left(\left(1-\rho^{2}\right) \partial_{\rho}-2 \rho \tau \partial_{\varepsilon}\right), J^{0}=\tau \partial_{\tau} \\
& P^{+}=\omega \tau, P^{-}=\omega\left(1-\rho^{2}\right) \tau^{-1}, P^{0}=\omega \rho .
\end{aligned}
$$


Let

$$
\begin{gathered}
f_{m}^{(\ell)}(\rho, \tau)=(\ell-m) ! \Gamma\left(m+\frac{1}{2}\right) C_{\ell-m}^{m+1 / 2}(\rho)(2 \tau)^{m} \\
m=\ell, \ell-1, \ell-2, \cdots
\end{gathered}
$$

where $C_{n}^{\nu}(z)$ is a Gegenbauer polynomial and $\ell \in C$ such that $\ell+\frac{1}{2}$ is not an integer. From the known recurrence relations obeyed by the $C_{n}^{\nu}(z),[11]$, and (5.12) it is easy to verify

$$
\begin{aligned}
& J^{0} f_{m}^{(\ell)}=m f_{m}^{(\ell)}, J^{ \pm} f_{m}^{(\ell)}=( \pm m-\ell) f_{m \pm 1}^{(\ell)} \\
& J \cdot J f_{m}^{(\ell)}=-\ell(\ell+1) f_{m}^{(\ell)} \\
& P^{0} f_{m}^{(\ell)}=\frac{\omega}{2 \ell+1} f_{m}^{(\ell+1)}+\frac{\omega(\ell+m)(\ell-m)}{2 \ell+1} f_{m}^{(\ell-1)} \\
& P^{ \pm} f_{m}^{(\ell)}=\frac{ \pm \omega}{2 \ell+1} f_{m \pm 1}^{(\ell+1)} \mp \frac{\omega(\ell \mp m)(\ell \mp m-1)}{2 \ell+1} f_{m \pm 1}^{(\ell-1)} .
\end{aligned}
$$

These relations determine the action of $\mathscr{E}(3)$ on the basis $\left\{f_{m}^{(\ell)}\right\}$ where $\ell=\ell_{0}, \ell_{0} \pm 1, \ell_{0} \pm 2, \cdots, m=\ell, \ell-1, \ell-2, \cdots$ and $2 \ell_{0}$ is not an integer. It is well-known that any entire function of $x$ can be expanded uniquely in a series of Gegenbauer polynomials $C_{n}^{\nu}(x), n=0,1,2, \cdots,(2 \nu \neq$ integer $)$, uniformly convergent in compact subsets of $C$, [23], page 238. Thus, one can exponentiate the $P$ and $J$ operators and compute the matrix elements of these operators in a $\left\{f_{m}^{(\ell)}\right\}$ basis. The rather complicated results are presented in [24]. One simple example is

$$
e^{\alpha P 0} f_{\ell}^{(\ell)}=\left(\frac{2}{\alpha}\right)^{\ell+1 / 2} \Gamma\left(\ell+\frac{1}{2}\right) \sum_{n=0}^{\infty} \frac{(\ell+n+1 / 2)}{n !} I_{\ell+n+1 / 2}(\alpha) f_{\ell}^{(\ell+n)}
$$

where $I_{\nu}(z)$ is a modified Bessel function, [11].

Now we consider the relationship between these results and solutions of the complex equation (0.1) in the spherical basis. Instead of the complex spherical coordinates $r, \theta, \varphi, 5]$, it is more convenient to use the equivalent separable coordinates

$$
\rho=-\cos \theta, \tau=-e^{i \varphi} \sin \theta, s=i r .
$$

In terms of these coordinates the symmetry operators for the Helmholtz equation are

$$
\begin{aligned}
& J^{+}=-\tau \partial_{\rho}, \quad J^{-}=\tau^{-1}\left(\left(1-\rho^{2}\right) \partial_{\rho}-2 \rho \tau \partial_{\tau}\right), \quad J^{0}=\tau \partial_{\tau} \\
& P^{+}=\tau \partial_{s}-\frac{\rho \tau}{s} \partial_{\rho}-\frac{\tau^{2}}{s} \partial_{\tau}, \\
& P^{-}=\frac{\left(1-\rho^{2}\right)}{\tau} \partial_{s}-\frac{\rho\left(1-\rho^{2}\right)}{s \tau} \partial_{\rho}+\frac{\left(\rho^{2}+1\right)}{s} \partial_{\tau},
\end{aligned}
$$




$$
P^{0}=\rho \partial_{s}+\frac{\left(1-\rho^{2}\right)}{s} \partial_{\rho}-\frac{\rho \tau}{s} \partial_{\tau}
$$

We search for a set of solutions $\left\{\Psi_{m}^{(\ell)}(x)\right\}$ of (0.1) which satisfy relations (5.14) when acted upon by symmetry operators (5.17). It is easily seen that

$$
\Psi_{m}^{(\ell)}(\boldsymbol{x})=S^{(\ell)}(s) f_{m}^{(\ell)}(\rho, \tau)
$$

where $S^{(\ell)}$ satisfies the recurrence formulas

$$
\left(\frac{d}{d s}-\frac{\ell}{s}\right) S^{(\ell)}(s)=\omega S^{(\ell+1)}(s), \quad\left(\frac{d}{d s}+\frac{\ell+1}{s}\right) S^{(\ell)}(s)=\omega S^{(\ell-1)}(s) .
$$

with independent solutions

$$
S^{(\ell)}(s)=(\omega s)^{-1 / 2}\left\{\begin{array}{l}
I_{\ell+1 / 2}(\omega s) \\
I_{-\ell-1 / 2}(\omega s) .
\end{array}\right.
$$

Choosing the upper solution we obtain the basis

$$
\Psi_{m}^{(\ell)}(s, \rho, \tau)=(\ell-m) ! \Gamma\left(m+\frac{1}{2}\right)(\omega s)^{-1 / 2} I_{\ell+1 / 2}(\omega s) C_{\ell-m}^{m+1 / 2}(\rho)(2 \tau)^{m} .
$$

It follows that the matrix elements giving the $E(3)$ group action which were computed for the $\left\{f_{m}^{(\ell)}\right\}$ basis are also valid for the $\left\{\Psi_{m}^{(\ell)}\right\}$ basis. For example, (5.15) leads to the addition theorem of Gegenbauer:

$$
\begin{aligned}
& I_{\ell+1 / 2}(s S)(2 S)^{-\ell-1 / 2}=\Gamma(\ell+1 / 2) \sum_{n=0}^{\infty}(\ell+n+1 / 2) I_{\ell+n+1 / 2}(s) \\
& I_{\ell+n+1 / 2}(\gamma) C_{n}^{\ell+1 / 2}(\rho), \quad S=\left(1+\gamma^{2} / s^{2}+2 \gamma \rho / s\right)^{1 / 2} .
\end{aligned}
$$

The complex sphere model can also be used to derive operational identities relating solutions of (0.1). For example, from (5.12), (5.13) we obtain the virtually trivial identity

$$
(\ell-m) ! C_{\ell-m}^{m+1 / 2}\left(\omega^{-1} P^{0}\right) f_{m}^{(m)}=f_{m}^{(\ell)}, \quad \ell-m=0,1, \cdots .
$$

However, for the model (5.17), (5.18) this identity assumes the non-trivial form

$$
C_{\ell-m}^{m+1 / 2}\left(\rho \partial_{s}+\frac{\left(1-\rho^{2}\right)}{s} \partial_{\rho}-\frac{\rho m}{s}\right) \frac{I_{m+1 / 2}(s)}{\sqrt{s}}=\frac{I_{\ell+1 / 2}}{\sqrt{s}} C_{\ell-m}^{m+1 / 2}(\rho) .
$$

Many other operational identities and addition theorems can be found in [24].

We present one example to show how Weisner's method in its general form can be used to derive expansions of solutions of the complex equa- 
tion (0.1) in terms of spherical waves. Consider the Laguerre polynomial solutions corresponding to the parabolic system 8]:

$$
\left(\left\{J_{1}, P_{2}\right\}-\left\{J_{2}, P_{1}\right\}\right) f=-2 i \lambda \omega f, \quad i J_{3} f=m f .
$$

Expressing these solutions in the coordinates (5.16) and expanding in the spherical basis we find

$$
\begin{aligned}
& e^{s \rho} L_{k}^{(m)}(-s(1+\rho)) L_{k}^{(m)}(s(1-\rho)) t^{m} \\
& \quad=\sum_{n=0}^{\infty} a_{n} s^{-m-1 / 2} I_{m+n+1 / 2}(s) C_{n}^{m+1 / 2}(\rho) t^{m}, \quad k=0,1,2, \ldots .
\end{aligned}
$$

The coefficients $a_{n}$ can be determined by setting $\rho=\alpha / s$ and letting $s=0$ to obtain

$$
2^{m+1 / 2} \Gamma\left(m+\frac{1}{2}\right) e^{\alpha}\left[L_{k}^{(m)}(-\alpha)\right]^{2}=\sum_{n=0}^{\infty} \frac{a_{n} \alpha^{n}}{n !(m+n+1 / 2)} .
$$

Use of the transformation formula for the ${ }_{1} F_{1}$ allows one to explicitly compute the coefficient of $\alpha^{n}$ on the left-hand side of this equation, with the result

$$
\begin{aligned}
a_{n}=\frac{2^{m+1 / 2}(m+n+1 / 2) \Gamma(m+1 / 2) \Gamma(m+k+1) \Gamma(m+k+n+1)}{(k !)^{2} \Gamma(m+1) \Gamma(m+n+1)} \\
\quad \times{ }_{3} F_{2}\left(\begin{array}{l}
-k,-m-n,-n \\
m+1,-m-k-n
\end{array} \mid 1\right) .
\end{aligned}
$$

For $k=0$ this expression reduces to (5.15).

\section{Section 6. $R$-Separable solutions of the Laplace equation.}

In addition to the eleven coordinate systems which separate for both the Helmholtz and Laplace equations there are other systems which $R$ separate for the Laplace equation (0.2) alone. The existence of these additional systems is related to the conformal symmetry of (0.2). All known systems for which (0.2) separates or $R$-separates have the property that the coordinate surfaces are orthogonal families of confocal cyclides or their degenerate limits. These surfaces, together with the corresponding Laplace separated equations, are described in detail in the classic book of Bôcher, [5]. Our primary aim is to provide a Lie algebraic characterization of the systems listed by Bôcher.

Each $R$-separable coordinate system $\{\mu, \nu, \rho\}$ with corresponding separated solutions 


$$
\Psi(\boldsymbol{x})=R^{1 / 2}(\mu, \nu, \rho) A(\mu) B(\nu) C(\rho)
$$

is associated with a two-dimensional subspace of commuting operators in $s o(4,1)^{2}$. If $S_{1}, S_{2}$ is a basis for this subspace, the separated solutions are characterized by the equations

$$
\Delta_{3} \Psi=0, S_{1} \Psi=\lambda_{1} \Psi, S_{2} \Psi=\lambda_{2} \Psi \text {. }
$$

Two systems are considered equivalent if one can be mapped to the other by a transformation of the conformal group. A list of the six $R$-separable systems is given in Table 2 .

TABLE 2. $R$-separable systems for the Laplace equation.

Commuting operators $S_{1}, S_{2}$

12]

$$
\begin{aligned}
S_{1}= & \frac{(a+1)}{4}\left(P_{2}+K_{2}\right)^{2} \\
& +\frac{(b+1)}{4}\left(P_{1}+K_{1}\right)^{2} \\
& +\frac{(a+b)}{4}\left(P_{3}+K_{3}\right)^{2} \\
& +J_{3}^{2}+b J_{2}^{2}+a J_{1}^{2}, \\
S_{2}= & \frac{a}{4}\left(P_{2}+K_{2}\right)^{2}+\frac{b}{4}\left(P_{1}+K_{1}\right)^{2} \\
& +\frac{a b}{4}\left(P_{3}+K_{3}\right)^{2}
\end{aligned}
$$

13] $S_{1}=2 \alpha J_{3}^{2}+\frac{(\alpha+1)}{2}\left\{P_{2}, K_{2}\right\}$

$$
\begin{aligned}
& +\frac{\beta}{2}\left(P_{2}^{2}-K_{2}^{2}\right)+\frac{\alpha}{2}\left\{P_{1}, K_{1}\right\} \\
& +\frac{\beta}{2}\left(K_{1}^{2}-P_{1}^{2}\right) \\
S_{2}= & \frac{\alpha}{2}\left\{P_{1}, K_{1}\right\}+\frac{\beta}{2}\left(P_{2}^{2}-K_{2}^{2}\right) \\
& +\left(\alpha^{2}+\beta^{2}\right) J_{3}^{2}
\end{aligned}
$$

14] $S_{1}=J_{3}^{2}$

$$
4 S_{2}=\left(P_{3}+K_{3}\right)^{2}-a\left(P_{3}-K_{3}\right)^{2}
$$

\section{Separable coordinates}

$$
\begin{aligned}
& x=R^{-1}\left[\frac{(\mu-a)(\nu-a)(\rho-a)}{(b-1)(a-1) a}\right]^{1 / 2} \\
& y=R^{-1}\left[\frac{(\mu-b)(\nu-b)(\rho-b)}{(a-b)(b-1) b}\right]^{1 / 2} \\
& z=R^{-1}\left[\frac{(\mu-1)(\nu-1)(\rho-1)}{(a-1)(b-1)}\right]^{1 / 2} \\
& R=1+\left[\frac{\mu \nu \rho}{a b}\right]^{1 / 2},
\end{aligned}
$$


15] $S_{1}=J_{3}^{2}$

$$
4 S_{2}=-4 a D^{2}-\left(P_{3}-K_{3}\right)^{2}
$$

16] $S_{1}=J_{3}^{2}$,

$$
2 S_{2}=\alpha\left\{P_{3}, K_{3}\right\}+\beta\left(K_{3}^{2}-P_{3}^{2}\right)
$$

17] $S_{1}=J_{3}^{2}$,

$$
S_{2}=\frac{1}{4}\left(P_{3}+K_{3}\right)^{2}
$$

$$
\begin{aligned}
R= & {\left[\frac{(\mu-a)(a-\rho)}{a(a-1)}\right]^{1 / 2} } \\
& -\left[\frac{(\mu-1)(1-\rho)}{a-1}\right]^{1 / 2},
\end{aligned}
$$

$x=R^{-1} \cos \varphi$

$y=R^{-1} \sin \varphi$

$$
\begin{aligned}
z & =R^{-1}\left[\frac{(\mu-a)(a-\rho)}{a(a-1)}\right]^{1 / 2} \\
R & =\left[\frac{\mu \rho}{a}\right]^{1 / 2}+\left[\frac{(\mu-1)(\rho-1)}{a(a-1)}\right]^{1 / 2},
\end{aligned}
$$

$x=R^{-1} \cos \varphi$

$y=R^{-1} \sin \varphi$

$$
z=R^{-1}\left[\frac{-\mu \rho}{a b}\right]^{1 / 2}
$$

$R=2 \operatorname{Re}\left[\frac{i(\rho-a)(\mu-a)}{a(a-b)}\right]^{1 / 2}$

$a=\vec{b}=\alpha+i \beta$

$x=R^{-1} \sinh \xi \cos \varphi$

$y=R^{-1} \sinh \xi \sin \varphi$

$z=R^{-1} \cos \Psi$

$R=\cosh \xi+\sin \Psi$

More specifically, for system 12] the parameters vary over the range $0<\rho<1<\nu<b<\mu<a$ and each factor in the separated solution satisfies

$$
\begin{gathered}
\left\{\sqrt{f(\xi)} \frac{d}{d \xi} \sqrt{f(\xi)} \frac{d}{d \xi}-\left(\frac{3 \xi^{2}}{16}+\frac{\lambda_{1}}{4} \xi+\frac{\lambda_{2}}{4}\right)\right\} A(\xi)=0, \\
f(\xi)=(\xi-a)(\xi-b)(\xi-1) \xi, \xi=\mu, \nu, \rho .
\end{gathered}
$$

This is the standard form of an equation with five elementary singularities, but very little is known about the solutions. For system 13] the parameters vary in the range $-\infty<\rho<0<\mu<1<\nu<\infty$ and the separated equations are (6.3) with $a=\bar{b}=\alpha+i \beta$.

For system 14] the parameters vary in the range $\mu>a>1, \rho<0$, $0 \leq \varphi<2 \pi$ and the solutions of (0.2) have the form $\Psi=R^{-1 / 2} E_{1}(\mu) E_{2}(\rho) e^{i m \varphi}$ where

$$
\left(4 \sqrt{p(\xi)} \frac{d}{d \xi} \sqrt{p(\xi)} \frac{d}{d \xi}+\left(\frac{1}{4}-m^{2}\right) \xi-\lambda\right) E_{j}(\xi)=0
$$




$$
\begin{aligned}
& j=1,2, \quad \xi=\mu, \rho, \quad \rho(\xi)=(\xi-a)(\xi-1) \xi, \\
& i J_{3} \Psi=m \Psi, \quad S_{2} \Psi=\lambda \Psi .
\end{aligned}
$$

If we set $\mu=s n^{2}(\alpha, k), \rho=s n^{2}(\beta, k)$ where $a=1 / k^{2}$ then we find

$$
\begin{aligned}
& x=R^{-1} \cos \varphi, \quad y=R^{-1} \sin \varphi, \quad z=k R^{-1} \operatorname{sn} \alpha \operatorname{sn} \beta \\
& R=k^{-1} k^{-1} d n \alpha d n \beta+i \operatorname{cn} \alpha \operatorname{cn} \beta, \quad \Psi=R^{1 / 2} \Lambda_{m-1 / 2}^{p}(\alpha, k) \Lambda_{m-1 / 2}^{p}(\beta, k) e^{i m \varphi}
\end{aligned}
$$

where $\Lambda_{n}^{p}(z, k)$ is a solution of the Lamé equation

$$
\frac{d^{2} \Lambda}{d z^{2}}+\left(h_{n}^{p}-n(n+1) k^{2} s n^{2}(z, k)\right) \Lambda=0 .
$$

The parameters $\alpha, \beta$ range over the intervals $\alpha \in\left[i K^{\prime}, i K^{\prime}+2 K\right], \beta \in[2 K$ $\left.-i K^{\prime}, 2 K+i K^{\prime}\right]$.

For system 15] the parameters vary in the range $1<\rho<a<\mu<\infty$, $0 \leq \varphi<2 \pi$ and the separation equations are (6.4). Making the same elliptic function substitutions as in the previous case, we find

$$
\begin{aligned}
& x=R^{-1} \cos \varphi, \quad y=R^{-1} \sin \varphi, \quad z=R^{-1} k k^{\prime} d n \alpha d n \beta \\
& R=k \operatorname{sn} \alpha \operatorname{sn} \beta+k k^{\prime-1} c n \alpha \operatorname{sn} \beta, \quad \Psi=R^{1 / 2} \Lambda_{m-1 / 2}^{p}(\alpha, k) \Lambda_{m-1 / 2}^{p}(\beta, k) e^{i m \varphi}
\end{aligned}
$$

where $\alpha \in\left[i K^{\prime}, i K^{\prime}+2 K\right], \beta \in\left[K, K+2 i K^{\prime}\right]$.

For system 16] the parameters satisfy $\mu>0, \rho<0,0 \leq \varphi<2 \pi$ and the separation equations are (6.4) with

$$
p(\xi)=(\xi-a)(\xi-b) \xi, \quad a=\bar{b}=\alpha+i \beta .
$$

Setting $\mu=s n^{2}(\alpha, t), \rho=s n^{2}(\beta, t)$ where $t=\left(s+i s^{\prime}\right)\left(s-i s^{\prime}\right)^{-1}, s^{2}=(|a|$

$-\operatorname{Re} a) / 2|a|$, we obtain solutions

$$
\begin{aligned}
& \Psi=R^{1 / 2} \Lambda_{m-1 / 2}^{p}(\alpha, t) \Lambda_{m-1 / 2}^{p}(\beta, t) e^{i m \varphi}, \\
& \alpha \in\left[-i K^{\prime}, i K^{\prime}\right], \quad \beta \in\left[2 K-i K^{\prime}, 2 K+i K^{\prime}\right] .
\end{aligned}
$$

Finally, for system 17], toroidal coordinates, the eigenfunctions have the form

$$
\begin{aligned}
& \Psi=(\cosh \xi+\sin \Psi)^{1 / 2} E(\xi) e^{i(\ell \Psi+m \varphi)} \\
& i J_{3} \Psi=m \Psi, \quad\left(P_{3}+K_{3}\right) \Psi=-2 i \ell \Psi \\
& {\left[\frac{1}{\sinh \xi} \frac{d}{d \xi} \sinh \xi \frac{d}{d \xi}+\left(\frac{1}{4}-\ell^{2}-\frac{m^{2}}{\sinh ^{2} \xi}\right)\right] E(\xi)=0 .}
\end{aligned}
$$

The associated Legendre functions $P_{\ell-1 / 2}^{m}(\cosh \xi), Q_{\ell-1 / 2}^{m}(\cosh \xi)$ provide a basis of solutions for this last equation. 
Systems 12]-16] are relatively intractible and only the toroidal system 17] has been widely used. The toroidal and spherical systems have much in common. Indeed, for the complex Laplace equation these two systems become equivalent under the complex conformal group. Bipolar coordinates, [7], page 108, are frequently used in connection with separation of variables for (0.2) but these coordinates are conformally equivalent to spherical coordinates. They are, however, inequivalent to spherical coordinates with respect to the more physical scale-Euclidean group, generated by $E(3)$ and dilatations.

Nine of the seventeen $R$-separable systems for (0.2) correspond to diagonalization of the operator $\left.\left.\left.\left.\left.J_{3}: 2\right], 5\right]-8\right], 14\right]-17\right]$. These special systems have the property that their eigenfunctions take the form $\Psi(x)=\Phi e^{i m \varphi}$, $i J_{3} \Psi=m \Psi$, where $\Phi$ is a function of the remaining two variables. If we substitute $\Psi$ into $(0.2)$ and factor out $e^{i m \varphi}$ we obtain a differential equation for $\Phi$ which in cylindrical coordinates is

$$
\left(\partial_{r r}+r^{-1} \partial_{r}-r^{-2} m^{2}+\partial_{z z}\right) \Phi(r, z)=0 .
$$

Equation (6.9) for fixed $m \geq 0$ is the equation of generalized axial-symmetric potential theory (GASPT). Its real symmetry algebra is isomorphic to $s \ell(2, R)$. Indeed, a basis is provided by the operators $K_{3}, P_{3}, D,(1.2)$, (1.16), with commutation relations

$$
\left[D, P_{3}\right]=P_{3}, \quad\left[D, K_{3}\right]=-K_{3}, \quad\left[K_{3}, P_{3}\right]=2 D,
$$

and from (1.25), iii), (6.9) can be written as

$$
\left(\frac{1}{2} P_{3}^{2}+\frac{1}{2} K_{3}^{2}-D^{2}\right) \Phi=\left(\frac{1}{4}+m^{2}\right) \Phi .
$$

It is shown in [25] that the space of symmetric second-order symmetry operators in the enveloping algebra of $s \ell(2, R)$, modulo the Casimir operaor $P_{3}^{2}+K_{3}^{2}-2 D^{2}$, decomposes into exactly nine orbit-types under the adjoint action of $S L(2, R)$. It is straightforward to check that the nine separable systems for (6.9) correspond exactly to these nine orbittypes.

\section{Section 7. Identities for solutions of the Laplace equation.}

Although it is not possible to find a Hilbert space model for solutions of (0.2) which leads to a unitary representation of the conformal group, we can still use Weisner's method and also construct non-Hilbert 
space models in a manner analogous to that in Section 5. Consider the expression

$$
\begin{gathered}
\Psi(\boldsymbol{x})=\int_{C_{1}} d \beta \int_{C_{2}} \frac{d t}{t} h(\beta, t) \exp \left[\frac{i x \beta}{2}\left(t+t^{-1}\right)\right. \\
\left.+\frac{y \beta}{2}\left(t-t^{-1}\right)-\beta z\right]=I(h),
\end{gathered}
$$

where $h$ is analytic on a domain in $C \times C$ which contains the integration contours $C_{1} \times C_{2}$ and is chosen such that $I(h)$ converges absolutely and arbitrary differentiation with respect to $x, y, z$ is permitted under the integral sign. It is easy to verify that for each such $h, \Psi=I(h)$ is a solution of the Laplace equation (0.2). Moreover, integrating by parts we find that the operators (1.2), (1.16) acting on the solution space of (0.2) correspond to the operators

$$
\begin{aligned}
P^{+} & =-\beta t, P^{-}=-\beta t^{-1}, P^{0}=-i \beta, D=\beta \partial_{\beta}+\frac{1}{2} \\
J^{+} & =i t \beta \partial_{\beta}-i t^{2} \partial_{t}, J^{-}=-\beta t^{-1} \partial_{\beta}-i \partial_{t}, J^{0}=t \partial_{t} \\
K^{+} & =t \beta^{-1}\left(\beta \partial_{\beta}-t \partial_{t}\right)\left(\beta \partial_{\beta}-t \partial_{t}-1\right), K^{-}=\left(t \beta^{-1}\right)\left(\beta \partial_{\beta}+t \partial_{t}\right)\left(\beta \partial_{\beta}+t \partial_{t}-1\right) \\
K^{0} & =i \beta^{-1}\left(\left(t \partial_{t}\right)^{2}-\left(\beta \partial_{\beta}\right)^{2}\right)
\end{aligned}
$$

where $J^{ \pm}=\mp J_{2}+i J_{1}, J^{0}=i J_{3}, K^{ \pm}=\mp K_{2}+i K_{1}, K^{0}=i K_{3}$, etc. Here we are assuming to $C_{1}, C_{2}$ and $h$ are chosen such that the boundary terms vanish for each integration by parts: $P^{ \pm} \Psi=I\left(P^{ \pm} h\right), J^{ \pm} \Psi=I\left(J^{ \pm} h\right)$, etc.

For our first example we choose $C_{1}, C_{2}$ as unit circles in the $\beta$ and $t$-plane, respectively, with centers at the origin and oriented in the counter-clockwise direction. Then for

$$
h(\beta, t)=\beta^{-\ell-1} j(t), \quad j(t)=\sum_{m=-\ell}^{\ell} a_{m} t^{m}, \quad \ell=0,1,2, \cdots
$$

we can evaluate the $\beta$-integral by residues to obtain

$$
\Psi(\boldsymbol{x})=I(h)=\frac{-2 \pi}{\ell !} \int_{0}^{2 \pi}[i x \cos \alpha+i y \sin \alpha-z]^{\ell} j\left(e^{i \alpha}\right) d \alpha .
$$

From (7.3), $h$ is an eigenfunction of $D$ with eigenvalue $-\ell-\frac{1}{2}$, so by (1.25), $J \cdot J \Psi=-\ell(\ell+1) \Psi$. Furthermore, $\Psi$ is a solution of (0.2) which is a homogeneous polynomial in $x, y, z$ of order $\ell$. In particular for $j(t)=t^{m}$, $-\ell \leq m \leq \ell$, we have $J^{0} \Psi=m \Psi$ so $\Psi$ must be a multiple of the solid harmonic $\rho^{\ell} Y_{\ell}^{m}(\theta, \varphi)$, (spherical coordinates). Evaluating the constant in the special case $\theta=0$ we find 


$$
\Psi_{\ell}^{m}=I\left(\beta^{-\ell-1} t^{m}\right)=\frac{16 \pi^{3}(-1)^{\ell+1} i^{m} \rho^{\ell}}{\sqrt{4 \pi(2 \ell+1)(\ell-m) !(\ell+m) !}} Y_{\ell}^{m}(\theta, \varphi) .
$$

Another example is provided by the contour $C_{2}$ in the $t$-plane and the contour $C_{1}^{\prime}$ which goes from $\beta=0$ to $+\infty$ along the positive real axis in the $\beta$-plane, and the analytic function $h(\beta, t)=\beta^{\ell} t^{m}, \ell=0,1,2, \ldots$, $m=\ell, \ell-1, \cdots,-\ell$. Here $\Psi^{\prime}=I^{\prime}(h)$ satisfies $D \Psi^{\prime}=\left(\ell+\frac{1}{2}\right) \Psi^{\prime}, J \cdot J \Psi^{\prime}=$ $-\ell(\ell+1) \Psi^{\prime}, J^{0} \Psi^{\prime}=m \Psi^{\prime}$ and it is easy to verify that

$$
\begin{aligned}
\Psi_{\ell}^{\prime m} & =I^{\prime}(h)=\frac{i \ell !}{\rho^{\ell+1}} \int_{0}^{2 \pi}[\cos \theta-i \sin \theta \cos (\varphi-\alpha)]^{-\ell-1} e^{i m \alpha} d \alpha \\
& =i^{1-m} \rho^{-\ell-1}\left[\frac{16 \pi^{3}(\ell-m) !(\ell+m) !}{2 \ell+1}\right]^{1 / 2} Y_{\ell}^{m}(\theta, \varphi),
\end{aligned}
$$

where $\rho, \theta, \varphi$ are spherical coordinates and $0 \leq \theta \leq \pi / 2$.

Now consider the equations

$$
\left(\left\{J_{1}, P_{2}\right\}-\left\{J_{2}, P_{1}\right\}\right) f=\lambda f, \quad J^{0} f=m f
$$

for the parabolic system 8]. In terms of model (7.2) the eigenfunctions are

$$
f_{\lambda, m}^{(8)}(\beta, t)=e^{-\lambda / 2 \beta} \beta^{-1} t^{m} .
$$

Setting $h=f_{\lambda, m}^{(8)}$ in (7.1) and choosing contours $C_{1}, C_{2}$ we find

$$
\begin{aligned}
\Psi_{\lambda, m}^{(8)} & =I\left(f_{\lambda, m}^{(8)}\right)=-2 \pi \int_{0}^{2 \pi} J_{0}\left[(-2 \lambda\{z-i x \cos \alpha-i y \sin \alpha\})^{1 / 2}\right] e^{i m \alpha} d \alpha \\
& =-4 \pi^{2} J_{m}(-i \sqrt{\lambda} \xi) J_{m}(\sqrt{\lambda} \eta) e^{i m \varphi}
\end{aligned}
$$

in terms of parabolic coordinates $\xi, \eta, \varphi$. As usual, the fact that variables separate enables us to compute the double integral. Using the contours $C_{1}^{\prime}, C_{2}$ we similarly obtain

$$
\begin{aligned}
\Psi_{\lambda, m}^{(8) \prime} & =I^{\prime}\left(f_{\lambda, m}^{(8)}\right) \\
& =2 \pi i^{m+1} e^{i m \varphi} \int_{0}^{\infty} J_{m}(\beta r) e^{-b z-\alpha / 2 \beta} \frac{d \beta}{\beta} \\
& =2 i \int_{0}^{2 \pi} K_{0}[\sqrt{2 \lambda} \sqrt{z-i x \cos \alpha-i y \sin \alpha}] e^{i m \alpha} d \alpha \\
& \left.=4 \pi i K_{m}(\sqrt{\lambda} \xi) I_{m}(i \sqrt{\lambda} \eta)\right) e^{i m \varphi}, \quad \lambda>0, \xi>|\eta|
\end{aligned}
$$

where $K_{\nu}(z)$ is a Macdonald function, [11]. The second and third equalities are obtained by performing only one of the integrations. Note that the 
second equality yields the expansion of our solution in terms of cylindrical waves. Similarly, performing the $t$-integration in (7.6) first, we find the expansion

$$
\Psi_{\ell}^{\prime m}=2 \pi i^{m+1} e^{i m \varphi} \int_{0}^{\infty} J_{m}(\beta r) e^{-\beta z} \beta^{\ell} d \beta, \quad z>0
$$

of a solid spherical harmonic in terms of cylindrical waves.

Applying the transformation $I$ (with contours $C_{1}, C_{2}$ ) to both sides of the identity

$$
f_{\lambda, m}^{(8)}(\beta, t)=t^{m} \sum_{\ell=0}^{\infty}\left(\frac{-\lambda}{2}\right)^{\ell} \frac{\beta^{-\ell-1}}{\ell !}
$$

one can easily obtain an expansion of the solutions $\Psi_{\lambda, m}^{(8)}$, (7.9), in terms of solid harmonics $\Psi_{\ell}^{m}$.

Corresponding to the oblate spheroidal system 7], the eigenvalue equations

$$
\left(\boldsymbol{J} \cdot \boldsymbol{J}+a^{2} P_{1}^{2}+a^{2} P_{2}^{2}\right) f=-\lambda f, \quad J^{0} f=m f
$$

in model (7.2) yield the eigenfunctions

$$
f_{\lambda, m}^{(7)}(\beta, t)=\beta^{-1 / 2} J_{\nu}(a \beta) t^{m}, \quad \nu^{2}=\lambda+\frac{1}{4} .
$$

Choosing the case where $m$ is a positive integer and $\nu=\ell+\frac{1}{2}, \ell>-1$, and applying the transformation $I^{\prime}$ (contours $C_{1}^{\prime}, C_{2}$ ) we find

$$
\begin{aligned}
\Psi_{\lambda, m}^{(7)}(\boldsymbol{x}) & =I^{\prime}\left(f_{\lambda, m}^{(7)}\right) \\
& =2 \pi i^{m+1} e^{i m \varphi} \int_{0}^{\infty} J_{m}(\beta r) J_{\nu}(a \beta) e^{-\beta z} \frac{d \beta}{\sqrt{\beta}} \\
& =\frac{2 \pi i^{m+1} \Gamma(m+\ell+1)}{\sqrt{a \cosh \eta}} e^{i m \varphi} P_{\ell}^{-m}(\cos \alpha) P_{m-1 / 2}^{-\ell-1 / 2}(\tanh \eta) \\
& 0<\alpha<\frac{\pi}{2}, 0<\eta
\end{aligned}
$$

where $\alpha, \eta, \varphi$ are oblate spheroidal coordinates. The second equality gives the expansion of our solution in terms of cylindrical waves. Again the integrals are rather easy to evaluate because one knows in advance that variables separate. In the case where $\nu=\ell+\frac{1}{2}, \ell=0,1, \cdots$, we can expand (7.11) as a power series in $\beta$ and apply $I$ term-by-term to obtain an expansion of (7.12) in solid spherical harmonics.

For the toroidal system 17] the eigenvalue equations 


$$
\left(P^{0}+K^{0}\right) f=2 \ell f, \quad J^{0} f=m f
$$

in model (7.2) yield the eigenfunctions

$$
f_{n, m}^{(17)}(\beta, t)=e^{-i \beta}(\beta t)^{m}{ }_{1} F_{1}\left(\begin{array}{c}
-n \\
2 m+1
\end{array} \mid 2 i \beta\right), \quad n=-\ell-m-\frac{1}{2} .
$$

We choose $n, m=0,1,2, \cdots$ and apply $I^{\prime}$ (contours $C_{1}^{\prime}, C_{2}$ ) to obtain

$$
\begin{aligned}
\Psi_{n, m}^{(17)}(\boldsymbol{x})= & I\left(f_{n, m}^{(17)}\right) \\
= & 2 \pi i^{m+1} e^{i m \varphi} \int_{0}^{\infty} e^{-\beta z-i \beta} J_{m}(r \beta) \beta^{m}{ }_{1} F_{1}\left(\begin{array}{c}
-n \\
2 m+1
\end{array} \mid 2 i \beta\right) d \beta \\
= & \pi\left(-\frac{1}{2}\right)^{m}(-1)^{n}(2 m) ! \sqrt{2 \cosh \xi+2 \sin \psi} \\
& \exp \left[i\left(m \varphi+\ell \psi+\frac{\pi}{4}\right)\right] P_{\ell-1 / 2}^{-m}(\cosh \xi) .
\end{aligned}
$$

An explicit computation yields

$$
\begin{aligned}
\exp \left(\alpha P_{3}\right) f_{n, m}^{(17)} & =\sum_{s=m}^{\infty} a_{n, m}^{s} \beta^{s} t^{m} \\
a_{n, m}^{s} & =\frac{(-\alpha-i)^{s-m}}{(s-m) !} F_{1} F_{1}\left(\begin{array}{c}
-n, m-s \\
2 m+1
\end{array} \mid(\alpha+i)^{-1}\right)
\end{aligned}
$$

so

$$
\exp \left(\alpha P_{3}\right) \Psi_{n, m}^{(17)}(\boldsymbol{x})=\sum_{s=m}^{\infty} a_{n, m}^{s} \Psi_{m}^{(s)}(\boldsymbol{x})
$$

is the expansion of this toroidal system solution in solid spherical harmonics. (The term-by-term integration used to derive (7.16) can easily be justified by the Lebesgue dominated convergence theorem.)

As the above examples indicate, our non-Hilbert space model permits us to derive integral representations and expansion formulas for the Laplace separable systems. The analysis for systems related to the Lamé and Whittaker-Hill equations proceeds in analogy with that in [16]. Of course, the number of examples can be greatly multiplied by choosing other contours in the $\beta$ and $t$-planes. In addition, the Hilbert space expansions for solutions of the wave equation $\left(\partial_{t t}-\Delta_{2}\right) \Psi=0$ and the $E P D$ equation can be re-interpreted as Laplace equation expansions by replacing $t$ with $i z$ for $z>0$, [26], [27].

The most useful functions for application of Weisner's method are those associated with the spherical system. These functions are char- 
acterized as eigenfunctions of $D$ and $J^{0}$. It is easy to check that in the model (7.2) the functions $g_{m}^{(\ell)}=i^{\ell-m} \beta^{\ell} t^{m}, \ell, m \in C$ satisfy the recurrence relations

$$
\begin{array}{rlrl}
J^{ \pm} g_{m}^{(\ell)} & =(-\ell \pm m) g_{m \pm 1}^{(\ell)}, & J^{0} g_{m}^{(\ell)} & =m g_{m}^{(\ell)} \\
P^{0} g_{m}^{(\ell)} & =-g_{m}^{(\ell+1)}, & & P^{ \pm} g_{m}^{(\ell)}=\mp g_{m \pm 1}^{(\ell+1)}, \\
D g_{m}^{(\ell)} & =\left(\ell+\frac{1}{2}\right) g_{m}^{(\ell)}, & K^{0} g_{m}^{(\ell)}=\left(\ell^{2}-m^{2}\right) g_{m}^{(\ell-1)} \\
K^{ \pm} g_{m}^{(\ell)} & =\mp(\ell \mp m)(\ell \mp m & -1) g_{m \pm 1}^{(\ell-1)} .
\end{array}
$$

From these relations one can compute the matrix elements of a conformal transformation in the $\left\{g_{m}^{(\ell)}\right\}$ basis. In the case where $\ell-m=$ $0,1,2, \cdots$ one can check that the corresponding basis of solutions for (0.2) which satisfies (7.17) is

$$
\begin{aligned}
& \Psi_{m}^{(\ell)}(w, t, \rho)=(\ell-m) ! \Gamma\left(m+\frac{1}{2}\right) C_{\ell-m}^{m+1 / 2}(w)(2 t)^{m}(\rho / i)^{-\ell-1}, \\
& w=\cos \theta=\frac{x_{3}}{\rho}, \quad t=\frac{x_{1}+i x_{2}}{\rho}, \quad \rho=\left[x_{1}^{2}+x_{2}^{2}+x_{3}^{2}\right]^{1 / 2} .
\end{aligned}
$$

Indeed, the relations (7.17) correspond to the known differential recurrence relations obeyed by the Gegenbauer polynomials $C_{n}^{\nu}(z)$. See [28] for derivations of addition theorems for the eigenfunctions (7.18) based on relations $(7.17)$.

The general Weisner method for (0.2) leads to expansions of the form

$$
\Psi(w, t, \rho)=\sum_{m, \ell} a_{m, \ell} C_{\ell-m}^{m+1 / 2}(w) t^{m}\left\{\begin{array}{l}
\rho^{\ell} \\
\rho^{-\ell-1}
\end{array}\right\}
$$

where $\Psi$ is an analytic solution of (0.2). If $J^{0} \Psi=m \Psi$ then $\Psi$ satisfies (6.9) and the double sum (7.19) reduces to a single sum on $\ell$. A very detailed discussion of possible Gegenbauer expansions in this special case is given by Viswanathan [29].

\section{REFERENCES}

[1] C. Boyer, E. G. Kalnins, and W. Miller, Jr., Lie theory and separation of variables. 6. The equation $i U_{t}+\Delta_{2} U=0$, J. Math. Phys., 16 (1975), 499-511.

[2] W. Miller, Jr., Symmetry, separation of variables and special functions, in Proceedings of the Advanced Seminar on Special Functions, Madison, Wisconsin, 1975, Academic Press, New York, 1975.

[ 3 ] P. Morse and H. Feshbach, Methods of Theoretical Physics, Part I, McGraw-Hill, New York, 1953. 
[4] A. A. Makarov, J. a. Smorodinsky, K. Valiev, and P. Winternitz, A systematic search for nonrelativistic systems with dynamical symmetries. Part I: The integrals of motion, Nuovo Cimento, 52A (1967), pp. 1061-1084.

[ 5 ] M. Bôcher, Die Reihenentwickelungen der Potentialtheorie, Leipzig, 1894.

[6 ] H. Bateman, Partial Differential Equations of Mathematical Physics, Cambridge University Press (First Edition, 1932), Reprinted 1969.

[ 7 ] H. Bateman, Electrical and Optical Wave-Motion, Dover 1955 (Reprint of 1914 Edition).

[ 8 ] N. Y. Vilenkin, Special Functions and the Theory of Group Representations, AMS Trans., Providence, Rhode Island, 1968 (English Transl.).

[9] W. Miller, Jr., Symmetry Groups and Their Applications, Academic Press, New York, 1972.

[10] F. Arscott, Periodic Differential Equations, Pergamon, Oxford, England, 1964.

[11] A. Erdélyi et al., Higher Transcendental Functions. Vols. 1 and 2, McGraw-Hill, New York, 1953.

[12] K. Urwin and F. Arscott, Theory of the Whittaker-Hill Equation, Proc. Roy. Soc. Edinb., A69 (1970), 28-44.

[13] W. Miller, Jr., Lie Theory and Separation of Variables II: Parabolic Coordinates, SIAM J. Math. Anal., 5 (1974), 822-836.

[14] W. Miller, Jr., Lie Theory and Special Functions, Academic Press, New York, 1968.

[15] J. Patera and P. Winternitz, A new basis for the representations of the rotation group. Lamé and Heun polynomials, J. Math. Phys., 14 (1973), 1130-1139.

[16] E. G. Kalnins and W. Miller, Jr., Lie theory and separation of variables. 4. The groups SO (2,1) and SO(3), J. Math. Phys., 15 (1974), 1263-1274.

[17] W. Miller, Jr., Symmetry and Separation of Variables for Linear Differential Equations, Addison-Wesley, Reading, Mass. (to appear).

[18] W. Miller, Jr., Lie Theory and Separation of Variables. 1. Parabolic Cylinder Coordinates, SIAM J. Math. Anal., 5 (1974), 626-643.

[19] H. Bucholz, The Confluent Hypergeometric Function, Springer-Verlag, New York, 1969.

[20] L. Weisner, Group-theoretic origin of certain generating functions, Pacific J. Math., 5 (1955), 1033-1039.

[21] E. G. Kalnins and W. Miller, Jr., Lie theory and separation of variables. 9. Orthogonal $R$-separable coordinate systems for the wave equation $\Psi_{t t}-\Delta_{2} \Psi=0$, J. Math. Phys. (to appear).

[22] E. G. Kalnins and W. Miller, Jr., Lie theory and separation of variables. 10. Nonorthogonal $R$-separable solutions of the wave equation $\partial_{t t} \Psi=\Delta_{2} \Psi$, J. Math. Phys. (to appear).

[23] F. W. Schafke, Einfuhrung in die Theorie der Speziellen Funktion der Mathematischen Physik, Springer-Verlag, Berlin, 1963.

[24] W. Miller, Jr., Special Functions and the Complex Euclidean Group in 3-Space. I, J. Math. Phys., 9 (1968), 1163-1175.

[25] P. Winternitz, I. Lukăc, and Y. Smorodinskii, Quantum numbers in the little groups of the Poincaré group, Soviet Physics JNP, 7 (1968), 139-145.

[26] E. G. Kalnins and W. Miller, Jr., Lie theory and separation of variables. 8. Semisubgroup coordinates for $\Psi_{t t}-\Delta_{2} \Psi=0$, J. Math. Phys. (to appear).

[27] E. G. Kalnins and W. Miller, Jr., Lie theory and separation of variables. 11. The Euler-Poisson-Darboux equation, J. Math. Phys (to appear).

[28] W. Miller, Jr., Special Functions and the Complex Euclidean Group in 3-Space. III, J. Math. Phys., 9 (1968), 1434-1444. 
[29] B. Viswanathan, Generating functions for ultraspherical functions, Can J. Math., 20 (1968), 120-134.

Universidad Nacional Autonoma de Mexico

University of Waikato

University of Minnesota 